\title{
Finite Element-Galerkin Approximation of the Eigenvalues and Eigenvectors of Selfadjoint Problems
}

\author{
By I. Babuska* and J. E. Osborn** \\ Dedicated to Professor Eugene Isaacson on the occasion of his 70 th birthday
}

\begin{abstract}
Refined estimates for finite element or, more generally, Galerkin approximations of the eigenvalues and eigenvectors of selfadjoint eigenvalue problems are presented. More specifically, refined results on the asymptotic behavior of the eigenvalue and eigenvector errors are proved. Both simple and multiple eigenvalues are treated.
\end{abstract}

1. Introduction. In this paper we establish some refined estimates for the approximation of the eigenvalues and eigenvectors of selfadjoint eigenvalue problems by finite element or, more generally, Galerkin methods. Suppose $\lambda$ is an eigenvalue of multiplicity $q$ of a selfadjoint problem and let $M(\lambda)$ denote the space of eigenvectors corresponding to $\lambda$. Denote by $\|\cdot\|_{B}$ the energy norm for the problem. Let $\left\{S_{h}\right\}_{0<h}$ be the family of finite-dimensional approximation spaces employed in the Galerkin method. $\lambda$ will be approximated from above by $q$ of the Galerkin approximate eigenvalues:

$$
\lambda \leq \lambda_{h, 1} \leq \cdots \leq \lambda_{h, q}, \quad \lambda \cong \lambda_{h, 1}, \ldots, \lambda_{h, q} .
$$

Let $u$, with $\|u\|_{B}=1$, denote an eigenvector corresponding to $\lambda$, and let $u_{h, 1}, \ldots$, $u_{h, q}$, with $\left\|u_{h, k}\right\|_{B}=1$, denote the Galerkin eigenvectors corresponding to $\lambda_{h, 1}, \ldots$, $\lambda_{h, q}$, respectively.

It is well known that

$$
\lambda_{h, k}-\lambda \leq C \sup _{\substack{u \in M(\lambda) \\\|u\|_{B}=1}} \inf _{\chi \in S_{h}}\|u-\chi\|_{B}^{2}, \quad k=1, \ldots, q,
$$

and that there is a $u_{k}=u_{k}(h) \in M(\lambda)$, with $\left\|u_{k}\right\|_{B}=1$, such that

$$
\left\|u_{h, k}-u_{k}\right\|_{B} \leq C \sup _{\substack{u \in M(\lambda) \\\|u\|_{B}=1}} \inf _{\chi \in S_{h}}\|u-\chi\|_{B}, \quad k=1, \ldots, q .
$$

In [7], [8] Chatelin proved the following refinements of (1.1) and (1.2):

$$
\begin{gathered}
\left\|u-E_{h} u\right\|_{B}=r_{h}^{(a)} \inf _{\chi \in S_{h}}\|u-\chi\|_{B} \quad \forall u \in M(\lambda), \\
\left\|u_{h, k}-E u_{h, k}\right\|_{B}=r_{h}^{(b)} \inf _{\chi \in S_{h}}\left\|E u_{h, k}-\chi\right\|_{B}, \quad k=1, \ldots, q,
\end{gathered}
$$

Received August 3, 1988.

1980 Mathematics Subject Classification (1985 Revision). Primary 65N15, 65N25, 65N30; Secondary 35P15.

* The work of this author was partially supported by the Office of Naval Research under Contract N00014-85-K-0169 and by the National Science Foundation under Grant DMS-85-16191.

** The work of this author was partially supported by the National Science Foundation under Grant DMS-84-10324. Part of the work was done while this author was supported as a visitor at the Institute for Mathematics and its Applications, University of Minnesota. 
and

$$
\left\|\left(\lambda_{h, k}-\lambda\right) / \lambda\right\|_{B}=r_{h}^{(c)} \inf _{\chi \in S_{h}}\left\|E u_{h, k}-\chi\right\|_{B}^{2}, \quad k=1, \ldots, q,
$$

where $E$ denotes the orthogonal projection of the energy space onto $M(\lambda)$ and $E_{h}$ the orthogonal projection onto $\operatorname{span}\left\{u_{h, 1}, \ldots, u_{h, q}\right\}$, and where $r_{h}^{(l)} \rightarrow 1$ as $h \rightarrow 0$, for $l=a, b, c$.

The purpose of this paper is twofold. The first is to establish an estimate for $\left|r_{h}^{(l)}-1\right|$. We show that

$$
\left|r_{h}^{(l)}-1\right| \leq d \eta^{2}(h)
$$

where $\eta(h)$ is a certain measure of the approximability property of $\left\{S_{h}\right\}$; for the definition of $\eta$ see Section 3. This is done in Section 4.

In [3] the authors established the estimate

$$
\lambda_{h, 1}-\lambda=C \inf _{\substack{u \in M(\lambda) \\\|u\|_{B}=1}} \inf _{\chi \in S_{h}}\|u-\chi\|_{B}^{2},
$$

which is an improvement over (1.1) and (1.3c) in the case of a multiple eigenvalue. [3] also contains estimates for $\lambda_{h, k}-\lambda, k=2, \ldots, q$, and for $\left\|u_{h, k}-u\right\|_{B}, k=$ $1, \ldots, q$, which are improvements of (1.1) and (1.3c) and of (1.2) and (1.3a,b), respectively. The second purpose of the paper is to present a simplified proof and an extension of the results in [3]. This is done in Section 5.

In Section 2 we give a precise statement of the class of eigenvalue problems and approximation methods we will consider. Section 3 contains some background information.

The second author would like to thank Professor Hans F. Weinberger for several helpful discussions on the topics in this paper.

2. Setting for the Problem. Suppose $H$ is a real Hilbert space with inner product $(\cdot, \cdot)$ and norm $\|\cdot\|$, respectively, and suppose we are given two symmetric bilinear forms $B(u, v)$ and $D(u, v)$ on $H \times H . B(u, v)$ is assumed to satisfy

$$
|B(u, v)| \leq C_{1}\|u\|\|v\| \quad \forall u, v \in H
$$

and

$$
C_{0}\|u\|^{2} \leq B(u, u) \quad \forall u \in H, \text { with } C_{0}>0 .
$$

It follows from (2.1) and (2.2) that $\|u\|_{B}=B(u, u)^{1 / 2}$ is equivalent to $\|u\|$. Regarding $D$, we assume

$$
0<D(u, u) \quad \forall 0 \neq u \in H
$$

and that

$$
\|u\|_{D}=D(u, u)^{1 / 2}
$$

is compact with respect to $\|\cdot\|$, i.e., from any sequence which is bounded in $\|\cdot\|$, one can extract a subsequence which is Cauchy in $\|\cdot\|_{D}$. For the remainder of this paper we will use $B(u, v)$ and $\|\cdot\|_{B}$ as the inner product and norm on $H$ and denote this space by $H_{B}$. 
We then consider the variationally formulated, selfadjoint eigenvalue problem

$$
\left\{\begin{array}{l}
\text { Seek } \lambda \text { (real) and } 0 \neq u \in H_{B} \text { satisfying } \\
B(u, v)=\lambda D(u, v) \quad \forall v \in H_{B} .
\end{array}\right.
$$

Under the assumptions we have made, (2.5) has a sequence of eigenvalues

$$
0<\lambda_{1} \leq \lambda_{2} \leq \cdots \nearrow+\infty
$$

and corresponding eigenvectors

$$
u_{1}, u_{2}, \ldots
$$

which can be chosen to satisfy

$$
B\left(u_{i}, u_{j}\right)=\lambda_{i} D\left(u_{i}, u_{j}\right)=\delta_{i j}, \quad i, j=1,2, \ldots
$$

The eigenvalues and eigenvectors satisfy the following well-known variational principles:

$$
\lambda_{k}=\min _{\substack{u \in H_{B} \\ B\left(u, u_{i}\right)=0 \\ i=1,2, \ldots, k-1}} \frac{B(u, u)}{D(u, u)}=\frac{B\left(u_{k}, u_{k}\right)}{D\left(u_{k}, u_{k}\right)}, \quad k=1,2, \ldots
$$

(the minimum principle)

and

$$
\lambda_{k}=\min _{\substack{V_{k} \subset H_{B} \\ \operatorname{dim} V_{k}=k}} \max _{u \in V_{k}} \frac{B(u, u)}{D(u, u)}=\max _{u \in U_{k}=\operatorname{span}\left(u_{1}, \ldots, u_{k}\right)} \frac{B(u, u)}{D(u, u)}, \quad k=1,2, \ldots
$$

(the minimum-maximum principle).

For any $\lambda_{k}$ we let

$$
M=M\left(\lambda_{k}\right)=\left\{u: u \text { is an eigenvector of (2.5) corresponding to } \lambda_{k}\right\} .
$$

We shall be interested in approximating the eigenpairs of $(2.5)$ by finite element or, more generally, Galerkin methods. Toward this end, we suppose we are given a (one-parameter) family $\left\{S_{h}\right\}_{0<h \leq 1}$ of finite-dimensional subspaces $S_{h} \subset H_{B}$, and we consider the eigenvalue problem

$$
\left\{\begin{array}{l}
\text { Seek } \lambda_{h} \text { (real), } 0 \neq u_{h} \in S_{h} \text { satisfying } \\
B\left(u_{h}, v\right)=\lambda_{h} D\left(u_{h}, v\right) \quad \forall v \in S_{h} .
\end{array}\right.
$$

The eigenpairs $\left(\lambda_{h}, u_{h}\right)$ of $(2.10)$ are then viewed as approximations to the eigenpairs $(\lambda, u)$ of $(2.5)$. (2.10) is called the Galerkin method determined by the subspaces $\left\{S_{h}\right\}$ for the approximation of the eigenvalues and eigenvectors of (2.5). We will also sometimes refer to problem (2.10) as the Galerkin approximation of problem (2.5). (2.10) has a sequence of eigenvalues

$$
0<\lambda_{h, 1} \leq \lambda_{h, 2} \leq \cdots \leq \lambda_{h, N}, \quad N=\operatorname{dim} S_{h},
$$

and corresponding eigenvectors

$$
u_{h, 1}, u_{h, 2}, \ldots, u_{h, N}
$$

which can be chosen to satisfy

$$
B\left(u_{h, i}, u_{h, j}\right)=\lambda_{h, i} D\left(u_{h, i}, u_{h, j}\right)=\delta_{i j}, \quad i, j=1, \ldots, N .
$$


The $\left(\lambda_{h, j}, u_{h, j}\right)$ are referred to as the approximate eigenpairs, while $\left(\lambda_{j}, u_{j}\right)$ are referred to as the exact eigenpairs of (2.5). Minimum and minimum-maximum principles analogous to (2.7) and (2.8) hold for problem (2.10); they are obtained from (2.7) and (2.8) by replacing $H_{B}$ by $S_{h}$ and letting $k=1, \ldots, N$. We will refer to them by $\left(2.7^{h}\right)$ and $\left(2.8^{h}\right)$, respectively. Using (2.7) and (2.8), together with $\left(2.7^{h}\right)$ and $\left(2.8^{h}\right)$, we see immediately that

$$
\lambda_{k} \leq \lambda_{h, k}, \quad k=1, \ldots, N=\operatorname{dim} S_{h} .
$$

We will assume that the family $\left\{S_{h}\right\}$ satisfies the approximability assumption

$$
\varepsilon_{u}(h)=\|u\|_{B}^{-1} \inf _{\chi \in S_{h}}\|u-\chi\|_{B} \rightarrow 0 \quad \text { as } h \rightarrow 0, \text { for each } u \in H_{B} .
$$

It follows from $(2.7),(2.8),\left(2.7^{h}\right),\left(2.8^{h}\right)$, and (2.13) that

$$
\lambda_{h, k} \rightarrow \lambda_{k} \quad \text { as } h \rightarrow 0, \text { for each } k .
$$

Finally we introduce

$$
\bar{u}_{j}=\sqrt{\lambda_{j}} u_{j}
$$

the exact eigenvectors normalized in $\|\cdot\|_{D}$, and

$$
\bar{u}_{h, j}=\sqrt{\lambda_{h, j}} u_{h, j},
$$

the approximate eigenvectors normalized in $\|\cdot\|_{D}$.

Throughout the paper, the specific eigenfunctions satisfying $(2.6)((2.11))$ will be denoted by $u_{j}\left(u_{h, j}\right)$. Thus the $u_{j}\left(u_{h, j}\right)$ are normalized in $\|\cdot\|_{B} ; \bar{u}_{j}\left(\bar{u}_{h, j}\right)$ denotes the same eigenvectors, renormalized in $\|\cdot\|_{D}$. When we denote an eigenpair by $(\lambda, u)$ we will not assume any particular normalization on $u$. $C, C_{i}, d$, and $d_{i}$ will denote generic constants.

3. Preliminary Results. In this section we present several preliminary results that will be used in the sequel. For further information on eigenvalue problems we refer the reader to [4], [8].

(a) An Identity Relating the Eigenvalue and Eigenvector Errors. Here we present an identity that relates the errors in eigenvalue and eigenvector approximation.

LEMMA 3.1. Suppose $(\lambda, u)$ is an eigenpair of (2.5), suppose $w$ is any vector in $H_{B}$ with $\|w\|_{D}=1$, and let $\lambda^{\prime}=B(w, w)$. Then

$$
\lambda^{\prime}-\lambda=\|w-u\|_{B}^{2}-\lambda\|w-u\|_{D}^{2} .
$$

Proof. By an easy calculation,

$$
\begin{aligned}
\|w-u\|_{B}^{2}-\lambda\|w-u\|_{D}^{2}= & \|w\|_{B}^{2}-2 B(w, u)+\|u\|_{B}^{2} \\
& -\lambda\|w\|_{D}^{2}+2 \lambda D(w, u)-\lambda\|u\|_{D}^{2} .
\end{aligned}
$$

Now

$$
B(v, u)=\lambda D(v, u) \quad \forall v \in H_{B}
$$

from which we get

$$
B(w, u)=\lambda D(w, u)
$$

and

$$
\|u\|_{B}^{2}=B(u, u)=\lambda D(u, u)=\lambda\|u\|_{D}^{2} .
$$


The result follows from (3.2)-(3.4) and the relations $\lambda^{\prime}=\|w\|_{B}^{2}$ and $1=\|w\|_{D}^{2}$.

(b) The Operators $T$ and $T_{h}$. Let

$$
H_{D}=\text { the completion of } H_{B} \text { with respect to }\|\cdot\|_{D} \text {. }
$$

$H_{D}$ is a Hilbert space with inner product $D$ and, since $\|\cdot\|_{D}$ is assumed to be compact with respect to $\|\cdot\|_{B}, H_{B}$ is compactly imbedded in $H_{D}$. (Alternatively, we could have assumed $H_{B} \subset H_{D}$, compactly, and let $D(u, v)$ be the inner product on $H_{D}$.)

From $H_{D}$ and $H_{B}$ construct the "negative space" $H_{-B}=H_{B}^{\prime}$, with norm $\|\cdot\|_{-B}$. Then $H_{D} \subset H_{-B}$ compactly, and for $v \in H_{B}, D(u, v)$ has a continuous extension to $u \in H_{-B}$ so that $D(u, v)$ is continuous on $H_{-B} \times H_{B}$. For $u \in H_{-B},\|u\|_{-B}=$ $\sup _{v \in H_{B}}|D(u, v)| /\|v\|_{B}$. For a complete discussion of this construction we refer to [5, pp. 31-39].

Next we introduce the operators $T, T_{h}: H_{-B} \rightarrow H_{B}$ defined by

$$
\begin{aligned}
& \left\{\begin{array}{l}
T f \in H_{B}, \\
B(T f, v)=D(f, v) \quad \forall v \in H_{B},
\end{array}\right. \\
& \left\{\begin{array}{l}
T_{h} f \in S_{h}, \\
B\left(T_{h} f, v\right)=D(f, v) \quad \forall v \in S_{h} .
\end{array}\right.
\end{aligned}
$$

$T$ and $T_{h}$ are the solution and approximate solution operators for the "boundary value" problem corresponding to the eigenvalue problem (2.5). It follows immediately from (2.1), (2.2), and the fact that $D(f, v)$ is continuous on $H_{-B} \times H_{B}$ that $T$ and $T_{h}$ are bounded from $H_{-B}$ to $H_{B}$. Since $H_{B}$ is compactly imbedded in $H_{D}$, and $H_{D}$ is compactly imbedded in $H_{-B}, T$ is compact from $H_{B}$ to $H_{B}$, from $H_{D}$ to $H_{D}$, and from $H_{-B}$ to $H_{-B} . T_{h}$ is, of course, also compact on $H_{B}, H_{D}$, and $H_{-B}$. It is easily seen that $T$ and $T_{h}$ are selfadjoint on $H_{D}$ and that $T$ is selfadjoint and positive definite on $H_{B}$ (with respect to $B(u, v)$ ). It is immediate that $T$ has eigenvalues

and eigenvectors

$$
\mu_{1}=\lambda_{1}^{-1} \geq \mu_{2}=\lambda_{2}^{-1} \geq \cdots \searrow 0
$$

$$
u_{1}, u_{2}, \ldots
$$

and that $T_{h}$ has eigenvalues

$$
\mu_{h, 1}=\lambda_{h, 1}^{-1} \geq \cdots \geq \mu_{h, N}=\lambda_{h, N}^{-1}, \quad N=\operatorname{dim} S_{h},
$$

and eigenvectors

$$
u_{h, 1}, \ldots, u_{h, N} \text {. }
$$

Let $P_{h}$ be the orthogonal projection of $H_{B}$ onto $S_{h}$; then from (3.6) we see that

$$
T_{h}=P_{h} T \text {. }
$$

Let

$$
\eta(h)=\left\|\left(I-P_{h}\right) T\right\|_{H_{D} \rightarrow H_{B}}=\left\|T-T_{h}\right\|_{H_{D} \rightarrow H_{B}}=\sup _{\substack{g \in H_{D} \\\|g\|_{D}=1}} \inf _{\chi \in S_{h}}\|T g-\chi\|_{B}
$$

and

$$
\nu(h)=\left\|\left(I-P_{h}\right) T\right\|_{H_{B} \rightarrow H_{B}}=\left\|T-T_{h}\right\|_{H_{B} \rightarrow H_{B}}=\sup _{\substack{g \in H_{B} \\\|g\|_{B}=1}} \inf _{\chi \in S_{h}}\|T g-\chi\|_{B}
$$


Several of the results in Sections 4 and 5 are stated in terms of the qualities of $\eta$ and $\nu$. We now present some properties of $\eta$ and $\nu$.

LEMMA 3.2. There are positive constants $C_{1}$ and $C_{2}$ such that

$$
C_{1} \nu(h) \leq \eta(h) \leq C_{2} \sqrt{\nu(h)} .
$$

Proof. Since $\|u\|_{D} \leq C\|u\|_{B} \forall u \in H_{B}$, we have $\nu(h) \leq C \eta(h)$, which is the first inequality in (3.9) with $C_{1}=C^{-1}$. Now consider the second inequality in (3.9). From (3.5) and (3.6) we have

$$
\|T f\|_{B} \leq\|f\|_{-B}, \quad\left\|T_{h} f\right\|_{B} \leq\|f\|_{-B}
$$

and hence

$$
\left\|T-T_{h}\right\|_{H_{-B} \rightarrow H_{B}} \leq 2,
$$

and from (3.8) we have

$$
\left\|T-T_{h}\right\|_{H_{B} \rightarrow H_{B}}=\nu(h) .
$$

We now note that $H_{-B}$ and $H_{B}$ are connected by a scale of Hilbert spaces. It thus follows from (3.10), (3.11), and a result on interpolation of linear operators $[5, \mathrm{pp}$. 240-242] that

$$
\eta(h)=\left\|T-T_{h}\right\|_{H_{D} \rightarrow H_{B}} \leq C 2^{1 / 2} \nu^{1 / 2}=C \nu(h)^{1 / 2},
$$

which is the second inequality in (3.9).

LEMMA 3.3. We have

$$
\lim _{h \rightarrow 0} \eta(h)=\lim _{h \rightarrow 0} \nu(h)=0 .
$$

Proof. Because of Lemma 3.2 it is sufficient to show that $\lim _{h \rightarrow 0} \nu(h)=0$. (2.13) implies that $P_{h} \rightarrow I$ pointwise on $H_{B}$ (in fact, (2.13) is equivalent to this result). Since $T: H_{B} \rightarrow H_{B}$ is compact, $T\left\{g \in H_{B}:\|g\|_{B}=1\right\}$ is relatively compact in $H_{B}$, and $\lim _{h \rightarrow 0} \nu(h)=0$ follows from the standard result that a family of operators that converges pointwise on a space converges uniformly on a relatively compact subset.

From Lemma 3.2 we have $\eta^{2}=O(\nu)$. It may happen that $\eta^{2}=o(\nu)$. This is shown by the following example.

Example. Let

$$
H_{B}=H_{0}^{1}(0,1), \quad B(u, v)=\int_{0}^{1} a(x) u^{\prime} v^{\prime} d x
$$

and

$$
D(u, v)=\int_{0}^{1} u v d x
$$

where $0<\alpha \leq a(x) \leq \beta<\infty$. $\left(H^{l}(0,1)\right.$ is the lth-order Sobolev space and $H_{0}^{1}(0,1)=\left\{u \in H^{1}(0,1): u(0)=u(1)=0\right\}$.) For $f \in L_{2}(0,1), u=T f$ is the solution of

$$
\left\{\begin{array}{l}
-\left(a(x) u^{\prime}\right)^{\prime}=f(x), \quad 0<x<1 \\
u(0)=u(1)=0
\end{array}\right.
$$


First suppose $S_{h}=$ the space of $C^{0}$ piecewise linear functions with mesh size $h$ that vanish at 0 and 1 and suppose $a(x)$ is smooth. Then we easily see that $\eta(h) \sim h$ and $\nu(h) \sim h$, so that $\eta^{2}=o(\nu)$. Next suppose $S_{h}=$ the space of $C^{0}$ piecewise quadratic functions vanishing at 0 and 1 . If $a(x)$ is smooth, we see that $\eta(h) \sim h$ and $\nu(h) \sim h^{2}$, so $\eta^{2} \sim \nu$. If, on the other hand, $a(x)$ is rough, specifically if $a(x)$ is such that $g \in H_{D}=L_{2}(0,1)$ implies $u=T f \in H^{2}(0,1)$, but $g \in H_{B}=H_{0}^{1}(0,1)$ does not, in general, imply $u \in H^{\alpha}(0,1)$ for $\alpha>2$, then $\eta \sim h$ and $\nu \sim h$, so $\eta^{2}=o(\nu)$.

From (2.13) we have

$$
\left\|\left(I-P_{h}\right) u\right\|_{B}=\varepsilon_{u}(h)\|u\|_{B} \rightarrow 0 \quad \forall u \in H_{B} .
$$

The usual duality argument (cf. Aubin [1], Nitsche [10], and Oganesjan-Rukhovets [11]) shows that $\left\|\left(I-P_{h}\right) u\right\|_{D} \leq C \eta(h)\left\|\left(I-P_{h}\right) u\right\|_{B}$ and $\left\|\left(I-P_{h}\right) u\right\|_{-B} \leq$ $C \nu(h)\left\|\left(I-P_{h}\right) u\right\|_{B}$. For the sake of completeness we include proofs of these results.

LEMMA 3.4. We have

$$
\left\|\left(I-P_{h}\right) u\right\|_{D} \leq \eta(h)\left\|\left(I-P_{h}\right) u\right\|_{B} \quad \forall u \in H_{B}
$$

and

$$
\left\|\left(I-P_{h}\right) u\right\|_{-B} \leq \nu(h)\left\|\left(I-P_{h}\right) u\right\|_{B} \quad \forall u \in H_{B} .
$$

Proof. Since $P_{h}$ is the orthogonal projection of $H_{B}$ onto $S_{h}$, we have

$$
B\left(\left(I-P_{h}\right) u, T g\right)=B\left(\left(I-P_{h}\right) u, T g-\chi\right) \quad \forall \chi \in S_{h},
$$

from which we get

$$
\left|B\left(\left(I-P_{h}\right) u, T g\right)\right| \leq\left\|\left(I-P_{h}\right) u\right\|_{B} \inf _{\chi \in S_{h}}\|T g-\chi\|_{B} .
$$

From (3.5), the symmetry of $D$ and $B$, and (3.14) we have

$$
\begin{aligned}
\left\|\left(I-P_{h}\right) u\right\|_{D} & =\sup _{\substack{g \in H_{D} \\
\|g\|_{D}=1}}\left|D\left(\left(I-P_{h}\right) u, g\right)\right|=\sup _{\substack{g \in H_{D} \\
\|g\|_{D}=1}}\left|B\left(\left(I-P_{h}\right) u, T g\right)\right| \\
& \leq \sup _{\substack{g \in H_{D} \\
\|g\|_{D}=1}} \inf _{\chi \in S_{h}}\|T g-\chi\|_{B}\left\|\left(I-P_{h}\right) u\right\|_{B} \leq \eta(h)\left\|\left(I-P_{h}\right) u\right\|_{B},
\end{aligned}
$$

which is (3.13a). Similarly,

$$
\begin{aligned}
\left\|\left(I-P_{h}\right) u\right\|_{-B} & =\sup _{\substack{g \in H_{B} \\
\|g\|_{B}=1}}\left|D\left(\left(I-P_{h}\right) u, g\right)\right| \\
& =\sup _{\substack{g \in H_{B} \\
\|g\|_{B}=1}} \inf _{\chi \in S_{h}}\|T g-\chi\|_{B}\left\|\left(I-P_{h}\right) u\right\|_{B}=\nu(h)\left\|\left(I-P_{h}\right) u\right\|_{B},
\end{aligned}
$$

which is $(3.13 \mathrm{~b})$.

(c) Preliminary Eigenvector Estimates. For $i=1,2, \ldots$ let $k_{i}$ be the lowest index of the $i$ th distinct eigenvalue of (2.5) and suppose $\lambda_{k_{i}}$ has multiplicity $q_{i}$. Let $E=E\left(\lambda_{k_{i}}\right)$ be the orthogonal projection of $H_{B}$ onto $M\left(\lambda_{k_{i}}\right)$ and let $E_{h}=E_{h}\left(\lambda_{k_{i}}\right)$ be the orthogonal projection of $H_{B}$ onto

$$
\begin{aligned}
M_{h}=M_{h}\left(\lambda_{k_{i}}\right)= & \text { the span of the eigenvectors of }(2.10) \\
& \text { corresponding to } \lambda_{h, k_{i}+j}, j=0, \ldots, q_{i}-1 .
\end{aligned}
$$


LEMMA 3.5. There is a constant $C_{i}$ such that

$$
\begin{array}{ll}
\left\|u-E_{h}\left(\lambda_{k_{i}}\right) u\right\|_{B} \leq C_{i}\left\|\left(I-P_{h}\right) u\right\|_{B} & \forall u \in M\left(\lambda_{k_{i}}\right), \\
\left\|u-E_{h}\left(\lambda_{k_{i}}\right) u\right\|_{D} \leq C_{i}\left\|\left(I-P_{h}\right) u\right\|_{D} & \forall u \in M\left(\lambda_{k_{i}}\right),
\end{array}
$$

and

$$
\left\|u-E_{h}\left(\lambda_{k_{i}}\right) u\right\|_{-B} \leq C_{i}\left\|\left(I-P_{h}\right) u\right\|_{-B} \quad \forall u \in M\left(\lambda_{k_{i}}\right) .
$$

Proof. Suppose the spaces $H_{B}, H_{D}$, and $H_{-B}$, the bilinear forms $B$ and $D$, and the operators $T, T_{h}, E$, and $E_{h}$ have been complexified in the usual manner. Let $\Gamma_{k_{i}}$ be a circle in the complex plane centered at $\mu_{k_{i}}=\lambda_{k_{i}}^{-1}$ and enclosing no other eigenvalues of $T$. Then for $h$ sufficiently small, $\mu_{h, k_{i}}=\lambda_{h, k_{i}}^{-1}, \ldots, \mu_{h, k_{i}+q_{i}-1}=$ $\lambda_{h, k_{i}+q_{i}-1}^{-1}$, but no other eigenvalues of $T_{h}$ are contained in $\Gamma_{k_{i}}$, and

$$
E\left(\lambda_{k_{i}}\right)=\frac{1}{2 \pi i} \int_{\Gamma_{k_{i}}}(z-T)^{-1} d z
$$

and

$$
E_{h}\left(\lambda_{k_{i}}\right)=\frac{1}{2 \pi i} \int_{\Gamma_{k_{i}}}\left(z-T_{h}\right)^{-1} d z .
$$

These are the usual formulas for the spectral projections associated with $T$ and $\mu_{k_{i}}$ and $T_{h}$ and $\mu_{h, k_{i}}, \ldots, \mu_{h, k_{i}+q_{i}-1}$, respectively (cf. [9, Section X1.9]).

Consider now the proof of (3.16a). Using (3.17) we have

$$
\begin{aligned}
\| u- & E_{h}\left(\lambda_{k_{i}}\right) u\left\|_{B}=\right\|\left[E\left(\lambda_{k_{i}}\right)-E_{h}\left(\lambda_{k_{i}}\right)\right] u \|_{B} \\
& =\left\|\frac{1}{2 \pi i} \int_{\Gamma_{k_{i}}}\left[(z-T)^{-1}-\left(z-T_{h}\right)^{-1}\right] u d z\right\|_{B} \\
& =\frac{1}{2 \pi}\left\|\int_{\Gamma_{k_{i}}}\left(z-T_{h}\right)^{-1}\left(T-T_{h}\right)(z-T)^{-1} u d z\right\|_{B} \\
& =\frac{1}{2 \pi}\left\|\int_{\Gamma_{k_{i}}}\left(z-T_{h}\right)^{-1}\left(T-T_{h}\right) \frac{u}{z-\mu_{k_{i}}} d z\right\|_{B} \underset{\sup }{ }\left\|\left(z-T_{h}\right)^{-1}\right\|_{H_{B} \rightarrow H_{B}} \frac{\left\|\left(T-T_{h}\right) u\right\|_{B}}{\operatorname{rad}\left(\Gamma_{k_{i}}\right)} \\
& \leq \frac{1}{2 \pi}\left[2 \pi \operatorname{rad}\left(\Gamma_{k_{i}}\right)\right] \operatorname{sur}_{z \in \Gamma_{k_{i}}} \\
& =\mu_{k_{i}} \sup _{z \in \Gamma_{k_{i}}}\left\|\left(z-T_{h}\right)^{-1}\right\|_{H_{B} \rightarrow H_{B}}\left\|\left(I-P_{h}\right) u\right\|_{B} \quad \forall u \in M\left(\lambda_{k_{i}}\right) .
\end{aligned}
$$

In the last inequality we used the relation $\left(T-T_{h}\right) u=\left(I-P_{h}\right) T u=\mu_{k_{i}}\left(I-P_{h}\right) u$. Now $\left\|T-T_{h}\right\|_{H_{B} \rightarrow H_{B}} \rightarrow 0$ implies

$$
C_{i}=\mu_{k_{i}} \sup _{\substack{z \in \Gamma_{k_{i}} \\ 0<h}}\left\|\left(z-T_{h}\right)^{-1}\right\|_{H_{B} \rightarrow H_{B}}<\infty,
$$

so we have established (3.16a).

Now consider the proof of $(3.16 \mathrm{~b})$. The above analysis is relative to the space $H_{B}$ (the integrals in (3.17) converge in the operator norm on $H_{B}$ and $\left.\left\|T-T_{h}\right\|_{H_{B} \rightarrow H_{B}} \rightarrow 0\right)$. Since $T$ and $T_{h}$ can also be considered on $H_{D}$ and 
$\left\|T-T_{h}\right\|_{H_{D} \rightarrow H_{D}} \rightarrow 0$, we can apply the same argument in $H_{D}$. Note that the formulas (3.17) will now define projections on $H_{D}$ which are extensions to $H_{D}$ of $E$ and $E_{h}$. We thus obtain (cf. (3.18))

$$
\left\|u-E_{h}\left(\lambda_{k_{i}}\right) u\right\|_{D} \leq \mu_{k_{i}} \sup _{\substack{z \in \Gamma_{k_{i}} \\ h>0}}\left\|\left(z-T_{h}\right)^{-1}\right\|_{H_{D} \rightarrow H_{D}}\left\|\left(I-P_{h}\right) u\right\|_{D} \quad \forall u \in M\left(\lambda_{k_{i}}\right)
$$

which is $(3.16 \mathrm{~b})$.

The proof of $(3.16 \mathrm{c})$ is similar.

Remark 3.1. It is essential in Lemma 3.5 that $h$ is sufficiently small, meaning small in comparison with the gap between $\lambda_{k_{i}}$ and $\lambda_{k_{i}-1}, \lambda_{k_{i}+1}$. If this gap is small, then it can happen that the approximate eigenfunction $u_{h, k_{i}}$ associated with $\lambda_{h, k_{i}}$ could be close to $u_{k_{i}-1}$ or $u_{k_{i}+1}$.

Lemma 3.5 is an eigenvector estimate since it provides an estimate for $u$ (an exact eigenvector) $-E_{h} u$ (a linear combination of approximate eigenvectors). We note that (2.13) and (3.16) imply that $E_{h}\left(\lambda_{k_{i}}\right): M\left(\lambda_{k_{i}}\right) \rightarrow M_{h}\left(\lambda_{k_{i}}\right)$ is one-toone and onto for $h$ sufficiently small.

We next prove a refinement of (3.16a) due to Chatelin [7], [8]. Inequality (3.16a) shows that

$$
\frac{\left\|u-E_{h}\left(\lambda_{k_{i}}\right) u\right\|_{B}}{\left\|u-P_{h} u\right\|_{B}}=O(1) \quad \forall u \in M\left(\lambda_{k_{i}}\right)
$$

Chatelin showed that

$$
\frac{\left\|u-E_{h}\left(\lambda_{k_{i}}\right) u\right\|_{B}}{\left\|u-P_{h} u\right\|_{B}} \rightarrow 1 \quad \text { as } h \rightarrow 0(\text { see }(1.3 \mathrm{a}))
$$

her argument, in fact, establishes

LEMMA 3.6 (CHATELIN). There is a constant $d_{i}$ such that

$$
1 \leq \frac{\left\|u-E_{h}\left(\lambda_{k_{i}}\right) u\right\|_{B}}{\left\|u-P_{h} u\right\|_{B}} \leq 1+d_{i} \nu(h) \quad \forall u \in M\left(\lambda_{k_{i}}\right)
$$

where $\nu(h)$ is defined in (3.8).

Proof. For the sake of completeness, and to establish the form of the bound in the second inequality in (3.19), we present a proof of this result.

Let $\bar{T}_{h}=P_{h} T P_{h}=T_{h} P_{h}$. Note that $\bar{T}_{h}$ and $T_{h}$ have the same nonzero eigenvalues, that $E_{h}\left(\lambda_{k_{i}}\right)$ commutes with $\bar{T}_{h}$, and that $\bar{T}_{h}$ is selfadjoint with respect to $B$. For $u \in M\left(\lambda_{k_{i}}\right)$,

$$
\left(\bar{T}_{h}-\mu_{h, k_{i}}\right) P_{h} u=P_{h} T\left(P_{h}-I\right) u+\left(\mu_{k_{i}}-\mu_{h, k_{i}}\right) P_{h} u
$$

and hence, since $E_{h}\left(\lambda_{k_{i}}\right)$ commutes with $\bar{T}_{h}$,

$$
\begin{aligned}
\left(\bar{T}_{h}-\mu_{h, k_{i}}\right)\left(I-E_{h}\left(\lambda_{k_{i}}\right)\right) P_{h} u= & \left(I-E_{h}\left(\lambda_{k_{i}}\right)\right) P_{h} T\left(P_{h}-I\right) u \\
& +\left(\mu_{k_{i}}-\mu_{h, k_{i}}\right)\left(I-E_{h}\left(\lambda_{k_{i}}\right)\right) P_{h} u .
\end{aligned}
$$

Let $Q$ be the orthogonal projection of $H_{B}$ onto $\mathscr{N}\left(\bar{T}_{h}\right)$, the null space of $\bar{T}_{h}$. Then, any $z \in \mathscr{R}\left(I-E_{h}\left(\lambda_{k_{i}}\right)\right)$, the range of $I-E_{h}\left(\lambda_{k_{i}}\right)$, can be written as

$$
z=\sum_{\substack{l=1 \\ l \neq k_{i}, \ldots, k_{i}+q_{i}-1}}^{N} B\left(z, u_{h, l}\right) u_{h, l}+Q z
$$


Here we have used the orthogonal decomposition

$$
\begin{aligned}
H_{B} & =\overline{\mathscr{R}\left(\bar{T}_{h}^{*}\right)} \oplus \mathscr{N}\left(\bar{T}_{h}\right)=\overline{\mathscr{R}\left(\bar{T}_{h}\right)} \oplus \mathscr{N}\left(\bar{T}_{h}\right) \\
& =\overline{\operatorname{span}\left\{u_{h, 1}, \ldots, u_{h, N}\right\}} \oplus \mathscr{N}\left(\bar{T}_{h}\right) \\
& =\operatorname{span}\left\{u_{h, 1}, \ldots, u_{h, N}\right\} \oplus \mathscr{N}\left(\bar{T}_{h}\right) .
\end{aligned}
$$

Thus,

$$
\left(\bar{T}_{h}-\mu_{h, k_{i}}\right) z=\sum_{\substack{l=1 \\ l \neq k_{i}, \ldots, k_{i}+q_{i}-1}}^{N} B\left(z, u_{h, l}\right)\left(\mu_{h, l}-\mu_{h, k_{i}}\right) u_{h, l}-\mu_{h, k_{i}} Q z
$$

and hence

$$
\begin{aligned}
\|\left(\bar{T}_{h}-\right. & \left.\mu_{h, k_{i}}\right) z \|_{B}^{2} \\
= & \sum_{\substack{l=1 \\
l \neq k_{i}, \ldots, k_{i}+q_{i}-1}}^{N}\left|B\left(z, u_{h, l}\right)\right|^{2}\left|\mu_{h, l}-\mu_{h, k_{i}}\right|^{2}+\left|\mu_{h, k_{i}}\right|^{2}\|Q z\|_{B}^{2} \\
\geq & \min \left\{\left|\mu_{h, j}-u_{h, k_{i}}\right|^{2}, j=1, \ldots, N, j \neq k_{i}, \ldots, k_{i}+q_{i}-1,\left|\mu_{h, k_{i}}\right|^{2}\right\} \\
& \times\left\{\begin{array}{l}
\sum_{\substack{l=1 \\
l \neq k_{i}, \ldots, k_{i}+q_{i}-1}}^{N}\left|B\left(z, u_{h, l}\right)\right|^{2}+\|Q z\|_{B}^{2} \\
\min \left\{\left|\mu_{h, k_{i}-1}-\mu_{h, k_{i}}\right|^{2},\left|\mu_{h, k_{i}+1}-\mu_{h, k_{i}}\right|^{2},\left|\mu_{h, k_{i}}\right|^{2}\right\}\|z\|_{B}^{2}, \quad i \geq 2, \\
\min \left\{\left|\mu_{h, k_{2}}-u_{h, k_{1}}\right|^{2},\left|u_{h, k_{1}}\right|^{2}\right\}\|z\|_{B}^{2}, \quad i=1 .
\end{array}\right.
\end{aligned}
$$

Since $\mu_{h, j} \rightarrow \mu_{j}$ (cf. (2.14)) for each $j$ as $h \rightarrow 0$,

$$
\begin{aligned}
& \left\{\begin{array}{l}
\min \left\{\left|\mu_{h, k_{i}-1}-\mu_{h, k_{i}}\right|^{2},\left|\mu_{h, k_{i}+1}-\mu_{h, k_{i}}\right|^{2},\left|\mu_{h, k_{i}}\right|^{2}\right\}, \quad i \geq 2, \\
\min \left\{\left|\mu_{h, k_{2}}-\mu_{h, k_{1}}\right|^{2},\left|\mu_{h, k_{1}}\right|^{2}\right\}, \quad i=1,
\end{array}\right. \\
& \rightarrow\left\{\begin{array}{l}
\min \left\{\left|\mu_{k_{i}-1}-\mu_{k_{i}}\right|^{2},\left|\mu_{k_{i}+1}-\mu_{k_{i}}\right|^{2},\left|\mu_{k_{i}}\right|^{2}\right\}, \quad i \geq 2, \\
\min \left\{\left|\mu_{k_{2}}-\mu_{k_{1}}\right|^{2},\left|\mu_{k_{1}}\right|^{2}\right\}, \quad i=1,
\end{array}\right. \\
& =\delta_{i}^{2} \quad \text { as } h \rightarrow 0 \text {, }
\end{aligned}
$$

from (3.21) we get

$$
\begin{aligned}
\left\|\left(\bar{T}_{h}-\mu_{h, k_{i}}\right) z\right\|_{B} \geq \delta_{i}\|z\|_{B} & \forall z \in \mathscr{R}\left(I-E_{h}\left(\lambda_{k_{i}}\right)\right) \text { and } \\
& \forall \text { small } h,
\end{aligned}
$$

where $\delta_{i}>0$ depends only on the gap between $\mu_{k_{i}}$ and $\mu_{k_{i}-1}, \mu_{k_{i}+1}$. Combining (3.20), (3.22), and the fact that $I-E_{h}\left(\lambda_{k_{i}}\right)$ and $P_{h}$ are orthogonal projections, we have

$$
\begin{aligned}
\|(I- & \left.E_{h}\left(\lambda_{k_{i}}\right)\right) P_{h} u \|_{B} \\
& \leq \delta_{i}^{-1}\left\|\left(I-E_{h}\left(\lambda_{k_{i}}\right)\right) P_{h} T\left(P_{h}-I\right) u+\left(\mu_{k_{i}}-\mu_{h, k_{i}}\right)\left(I-E_{h}\left(\lambda_{k_{i}}\right)\right) P_{h} u\right\|_{B} \\
& \leq \delta_{i}^{-1}\left\{\left\|T\left(P_{h}-I\right)^{2} u\right\|_{B}+\left|\mu_{k_{i}}-\mu_{h, k_{i}}\right|\left\|\left(I-E_{h}\left(\lambda_{k_{i}}\right)\right) P_{h} u\right\|_{B}\right\},
\end{aligned}
$$

from which we get

$$
\begin{aligned}
\left\|\left(I-E_{h}\left(\lambda_{k_{i}}\right)\right) P_{h} u\right\|_{B} & \leq d_{i}\left\|T\left(P_{h}-I\right)\right\|_{H_{B} \rightarrow H_{B}}\left\|\left(P_{h}-I\right) u\right\|_{B} \\
& =d_{i}\left\|\left(P_{h}-I\right) T\right\|_{H_{B} \rightarrow H_{B}}\left\|\left(P_{h}-I\right) u\right\|_{B} .
\end{aligned}
$$


In the last equality we used the fact that $\left(P_{h}-I\right)$ and $T$ are selfadjoint and that the norm of an operator and its adjoint are equal.

(3.23) implies

$$
\begin{gathered}
\|\|\left(I-E_{h}\left(\lambda_{k_{i}}\right) P_{h}\right) u\left\|_{B}-\right\|\left(I-P_{h}\right) u\left\|_{B} \mid \leq\right\|\left(I-E_{h}\left(\lambda_{k_{i}}\right)\right) P_{h} u \|_{B} \\
\quad \leq d_{i}\left\|\left(P_{h}-I\right) T\right\|_{H_{B} \rightarrow H_{B}}\left\|\left(P_{h}-I\right) u\right\|_{B},
\end{gathered}
$$

and hence

$$
\left|\frac{\left\|\left(I-E_{h}\left(\lambda_{k_{i}}\right) P_{h}\right) u\right\|_{B}}{\left\|\left(P_{h}-I\right) u\right\|_{B}}-1\right| \leq d_{i}\left\|\left(P_{h}-I\right) T\right\|_{H_{B} \rightarrow H_{B}} .
$$

We easily see that

$$
\left\|\left(I-P_{h}\right) u\right\|_{B} \leq\left\|\left(I-E_{h}\left(\lambda_{k_{i}}\right)\right) u\right\|_{B} \leq\left\|\left(I-E_{h}\left(\lambda_{k_{i}}\right) P_{h}\right) u\right\|_{B},
$$

and thus

$$
1 \leq \frac{\left\|\left(I-E_{h}\left(\lambda_{k_{i}}\right)\right) u\right\|_{B}}{\left\|\left(P_{h}-I\right) u\right\|_{B}} \leq \frac{\left\|\left(I-E_{h}\left(\lambda_{k_{i}}\right) P_{h}\right) u\right\|_{B}}{\left\|\left(I-P_{h}\right) u\right\|_{B}} .
$$

Combining (3.24) and (3.25), we have

$$
\begin{aligned}
0 & \leq \frac{\left\|\left(I-E_{h}\left(\lambda_{k_{i}}\right)\right) u\right\|_{B}}{\left\|\left(P_{h}-I\right) u\right\|_{B}}-1 \leq \frac{\left\|\left(I-E_{h}\left(\lambda_{k_{i}}\right) P_{h}\right) u\right\|_{B}}{\left\|\left(P_{h}-I\right) u\right\|_{B}}-1 \\
& \leq d_{i}\left\|\left(P_{h}-I\right) T\right\|_{H_{B} \rightarrow H_{B}} \quad \forall u \in M\left(\lambda_{k_{i}}\right) .
\end{aligned}
$$

Recalling that $\left\|\left(P_{h}-I\right) T\right\|_{H_{B} \rightarrow H_{B}}=\nu(h)$, we obtain the desired result.

Remark 3.2. (3.19) should be compared with (4.20), which provides a stronger estimate for certain special $u$ 's in $M\left(\lambda_{k_{i}}\right)$.

Lemmas 3.5 and 3.6 show that starting from any exact eigenvector $u$ we can construct $E_{h}\left(\lambda_{k_{i}}\right) u$, a linear combination of approximate eigenvectors that is close to $u$. One can also start with an approximate eigenvector and construct a close exact eigenvector. We present another result of Chatelin [7], [8]; see (1.3b).

LEMMA 3.7 (CHATELIN). There is a constant $d_{i}$ such that

$$
1 \leq \frac{\left\|u_{h, j}-E\left(\lambda_{k_{i}}\right) u_{h, j}\right\|_{B}}{\left\|P_{h} E\left(\lambda_{k_{i}}\right) u_{h, j}-E\left(\lambda_{k_{i}}\right) u_{h, j}\right\|_{B}} \leq 1+d_{i} \nu(h), \quad j=k_{i}, \ldots, k_{i}+q_{i}-1 \text {. }
$$

Proof. Observing that

$$
E\left(\lambda_{k_{i}}\right)-E_{h}\left(\lambda_{k_{i}}\right) P_{h}=\left(E\left(\lambda_{k_{i}}\right)-E_{h}\left(\lambda_{k_{i}}\right)\right) P_{h}+E\left(\lambda_{k_{i}}\right)\left(I-P_{h}\right),
$$

we obtain

$$
\begin{aligned}
\left\|E\left(\lambda_{k_{i}}\right)-E_{h}\left(\lambda_{k_{i}}\right) P_{h}\right\|_{H_{B} \rightarrow H_{B}} \leq & \left\|E\left(\lambda_{k_{i}}\right)-E_{h}\left(\lambda_{k_{i}}\right)\right\|_{H_{B} \rightarrow H_{B}} \\
& +\left\|E\left(\lambda_{k_{i}}\right)\left(I-P_{h}\right)\right\|_{H_{B} \rightarrow H_{B}} .
\end{aligned}
$$

We easily see that

$$
\begin{aligned}
\left\|E\left(\lambda_{k_{i}}\right)\left(I-P_{h}\right)\right\|_{H_{B} \rightarrow H_{B}} & =\left\|\left(I-P_{h}\right) E\left(\lambda_{k_{i}}\right)\right\|_{H_{B} \rightarrow H_{B}} \\
& =\sup _{\substack{u \in H_{B} \\
\|u\|_{B}=1}}\left\|\left(I-P_{h}\right) E\left(\lambda_{k_{i}}\right) u\right\|_{B} \\
& =\lambda_{k_{i}} \sup _{\substack{u \in H_{B} \\
\|u\|_{B}=1}}\left\|\left(I-P_{h}\right) T E\left(\lambda_{k_{i}}\right) u\right\|_{B} \leq \lambda_{k_{i}} \nu(h),
\end{aligned}
$$


and by a slight modification of estimate (3.18) we have

$$
\left\|E\left(\lambda_{k_{i}}\right)-E_{h}\left(\lambda_{k_{i}}\right)\right\|_{H_{B} \rightarrow H_{B}} \leq C \nu(h)
$$

Thus,

$$
\left\|E\left(\lambda_{k_{i}}\right)-E_{h}\left(\lambda_{k_{i}}\right) P_{h}\right\|_{H_{B} \rightarrow H_{B}} \leq C \nu(h)
$$

Next note that

$$
\left\{I-\left[E_{h}\left(\lambda_{k_{i}}\right) P_{h}-E\left(\lambda_{k_{i}}\right)\right]\right\}\left(u_{h, j}-E\left(\lambda_{k_{i}}\right) u_{h, j}\right)=\left[E_{h}\left(\lambda_{k_{i}}\right) P_{h}-I\right] E\left(\lambda_{k_{i}}\right) u_{h, j} .
$$

Hence, using (3.24) and (3.27), we have

$$
\begin{aligned}
\left\|u_{h, j}-E\left(\lambda_{k_{i}}\right) u_{h, j}\right\|_{B} \leq & \left\|\left\{I-\left[E_{h}\left(\lambda_{k_{i}}\right) P_{h}-E\left(\lambda_{k_{i}}\right)\right]\right\}^{-1}\right\|_{H_{B} \rightarrow H_{B}} \\
& \times\left\|\left[E_{h}\left(\lambda_{k_{i}}\right) P_{h}-I\right] E\left(\lambda_{k_{i}}\right) u_{h, j}\right\|_{B} \\
& \leq \frac{\left\|\left[E_{h}\left(\lambda_{k_{i}}\right) P_{h}-I\right] E\left(\lambda_{k_{i}}\right) u_{h, j}\right\|_{B}}{1-\left\|E_{h}\left(\lambda_{k_{i}}\right) P_{h}-E\left(\lambda_{k_{i}}\right)\right\|_{H_{B} \rightarrow H_{B}}} \\
& \leq \frac{\left(1+d_{i} \nu\right)\left\|\left(P_{h}-I\right) E\left(\lambda_{k_{i}}\right) u_{h, j}\right\|_{B}}{1-C \nu}
\end{aligned}
$$

which implies the second inequality in (3.26). The first is immediate.

(d) Relation Between Eigenvector Error in $\|\cdot\|_{B},\|\cdot\|_{D}$, and $\|\cdot\|_{-B}$. In Subsection 3. (b) we noted that $\left\|\left(I-P_{h}\right) u\right\|_{D} \leq \eta(h)\left\|\left(I-P_{h}\right) u\right\|_{B}$ and $\left\|\left(I-P_{h}\right) u\right\|_{-B} \leq$ $\nu(h)\left\|\left(I-P_{h}\right) u\right\|_{B}$. In this subsection we establish similar results for the eigenvector error.

For $i=1,2, \ldots$ and $j=k_{i}, \ldots, k_{i}+q_{i}-1$, let $\bar{u}_{j}^{h} \in M\left(\lambda_{k_{i}}\right)$ satisfy $E_{h}\left(\lambda_{k_{i}}\right) \bar{u}_{j}^{h}=$ $\bar{u}_{h, j}$. We know from the discussion in Subsection 3.(c) that $\bar{u}_{j}^{h}$ exists and is unique for $h$ small. From (3.13a) and (3.16b) we have

$$
\begin{aligned}
\left\|\bar{u}_{j}^{h}-\bar{u}_{h, j}\right\|_{D} & =\left\|\bar{u}_{j}^{h}-E_{h}\left(\lambda_{k_{i}}\right) \bar{u}_{j}^{h}\right\|_{D} \leq C_{i} \eta(h)\left\|\left(I-P_{h}\right) \bar{u}_{j}^{h}\right\|_{B} \\
& \leq C_{i} \eta(h)\left\|\bar{u}_{j}^{h}-\bar{u}_{h, j}\right\|_{B},
\end{aligned}
$$

or

$$
\frac{\left\|\bar{u}_{j}^{h}-\bar{u}_{h, j}\right\|_{D}}{\left\|\bar{u}_{j}^{h}-\bar{u}_{h, j}\right\|_{B}} \leq C_{i} \eta(h) .
$$

It follows immediately (by scaling) that

$$
\frac{\left\|u_{j}^{h}-u_{h, j}\right\|_{D}}{\left\|u_{j}^{h}-u_{h, j}\right\|_{B}} \leq C_{i} \eta(h),
$$

where $u_{j}^{h} \in M\left(\lambda_{k_{i}}\right)$ satisfies $E_{h}\left(\lambda_{k_{i}}\right) u_{j}^{h}=u_{h, j}$. (Recall that $\left\|\bar{u}_{j, h}\right\|_{D}=1$ and $\left\|u_{j, h}\right\|_{B}=1$.) Similarly, from (3.13b) and (3.16c) we get

$$
\frac{\left\|\bar{u}_{j}^{h}-\bar{u}_{h, j}\right\|_{-B}}{\left\|\bar{u}_{j}^{h}-\bar{u}_{h, j}\right\|_{B}} \leq C_{i} \nu(h)
$$

and

$$
\frac{\left\|u_{j}^{h}-u_{h, j}\right\|_{-B}}{\left\|u_{j}^{h}-u_{h, j}\right\|_{B}} \leq C_{i} \nu(h) .
$$


By Lemma 3.1 we know that

$$
\begin{aligned}
\lambda_{h, j}-\lambda_{k_{i}} & =\left\|u-\bar{u}_{h, j}\right\|_{B}^{2}-\lambda_{k_{i}}\left\|u-\bar{u}_{h, j}\right\|_{D}^{2} \\
& =\left\|u-\bar{u}_{h, j}\right\|_{B}^{2}\left\{1-\lambda_{k_{i}} \frac{\left\|u-\bar{u}_{h, j}\right\|_{D}^{2}}{\left\|u-\bar{u}_{h, j}\right\|_{B}^{2}}\right\} \quad \forall u \in M\left(\lambda_{k_{i}}\right) .
\end{aligned}
$$

As $u$ varies over $M\left(\lambda_{k_{i}}\right)$ it is clear from (3.30) that $\left\|u-\bar{u}_{h, j}\right\|_{D}^{2} /\left\|u-\bar{u}_{h, j}\right\|_{B}^{2}$ is minimized for that $\bar{u}_{0}$ that minimizes $\left\|u-\bar{u}_{h, j}\right\|_{B}^{2}$, namely for $u_{0}=E\left(\lambda_{k_{i}}\right) \bar{u}_{h, j}$. Thus we have

$$
\frac{\left\|E\left(\lambda_{k_{i}}\right) \bar{u}_{h, j}-\bar{u}_{h, j}\right\|_{D}}{\left\|E\left(\lambda_{k_{i}}\right) \bar{u}_{h, j}-\bar{u}_{h, j}\right\|_{B}} \leq \frac{\left\|\bar{u}_{j}^{h}-\bar{u}_{h, j}\right\|_{D}}{\left\|\bar{u}_{j}^{h}-\bar{u}_{h, j}\right\|_{B}} \leq C_{i} \eta(h) .
$$

We, of course, also get

$$
\frac{\left\|E\left(\lambda_{k_{i}}\right) u_{h, j}-u_{h, j}\right\|_{D}}{\left\|E\left(\lambda_{k_{i}}\right) u_{h, j}-u_{h, j}\right\|_{B}} \leq C_{i} \eta(h)
$$

Estimates (3.31) are similar to (3.28), but involve a different pairing of approximate and exact eigenvectors.

Remark 3.3. Pierce and Varga [12] proved eigenvector estimates in $\|\cdot\|_{D}$, and Bramble and Osborn [6] established them in $\|\cdot\|_{-B}$.

\section{Precise Asymptotic Estimates for the Eigenvalue and Eigenvector}

Error. In this section we use the notation introduced in Subsection 3.(c), i.e., we let $k_{i}$ be the lowest index of the $i$ th distinct eigenvalue of (2.5) and assume $\lambda_{k_{i}}$ has multiplicity $q_{i}$.

(a) The Eigenvalue Error. For $i=1,2, \ldots$ and $j=k_{i}, \ldots, k_{i}+q_{i}-1$ fixed, Chatelin [7], [8] has shown that

$$
\frac{\left(\lambda_{h, j}-\lambda_{k_{i}}\right) / \lambda_{k_{i}}}{\left\|\left(I-P_{h}\right) E\left(\lambda_{k_{i}}\right) u_{h, j}\right\|_{B}^{2} /\left\|E\left(\lambda_{k_{i}}\right) u_{h, j}\right\|\left[C_{B}^{2}\right.} \rightarrow 1 \quad \text { as } h \rightarrow 0 \text { (cf. (1.3c)). }
$$

We now prove a refinement of (4.1) (cf. (1.3c) and (1.4)).

THEOREM 4.1. For $i=1,2, \ldots$ there is a constant $d_{i}$ such that

$$
\left|\frac{\left(\lambda_{h, j}-\lambda_{k_{i}}\right) / \lambda_{k_{i}}}{\left\|\left(I-P_{h}\right) E\left(\lambda_{k_{i}}\right) u_{h, j}\right\|_{B}^{2} /\left\|E\left(\lambda_{k_{i}}\right) u_{h, j}\right\|_{B}^{2}}-1\right| \leq d_{i} \eta^{2}(h),
$$

$j=k_{i}, \ldots, k_{i}+q_{i}-1$, where $\eta(h)$ is defined in (3.7).

Proof. Let $u=E\left(\lambda_{k_{i}}\right) u_{h, j}$. We have

$$
\begin{aligned}
\left(\mu_{k_{i}}\right. & \left.-\mu_{h, j}\right) B\left(u, u_{h, j}\right)=B\left(T u, u_{h, j}\right)-B\left(u, T_{h} u_{h, j}\right) \\
& =B\left(u,\left(T-T_{h}\right) u_{h, j}\right)=B\left(T\left(I-P_{h}\right) u, u_{h, j}\right) \\
& =B\left(T\left(I-P_{h}\right) u, u\right)+B\left(T\left(I-P_{h}\right) u, u_{h, j}-u\right) \\
& =B\left(T\left(I-P_{h}\right)^{2} u, u\right)+B\left(T\left(I-P_{h}\right) u, u_{h, j}-u\right) \\
& =\mu_{k_{i}} B\left(\left(I-P_{h}\right) u,\left(I-P_{h}\right) u\right)+D\left(\left(I-P_{h}\right) u, u_{h, j}-u\right) .
\end{aligned}
$$

Using the fact that $B\left(u, u_{h, j}\right)=B\left(u, E\left(\lambda_{k_{i}}\right) u_{h, j}\right)=\|u\|_{B}^{2}$, (4.3) can be written as

$$
\frac{\lambda_{h, j}-\lambda_{k_{i}}}{\lambda_{h, j} \lambda_{k_{i}}}\|u\|_{B}^{2}=\frac{1}{\lambda_{k_{i}}}\left\|\left(I-P_{h}\right) u\right\|_{B}^{2}+D\left(\left(I-P_{h}\right) u, u_{h, j}-u\right) .
$$


Dividing by $\left\|\left(I-P_{h}\right) u\right\|_{B}^{2}$, multiplying by $\lambda_{h, j}$, and subtracting 1 from both sides, we find

$$
\frac{\left(\lambda_{h, j}-\lambda_{k_{i}}\right) / \lambda_{k_{i}}}{\left\|\left(I-P_{h}\right) u\right\|_{B}^{2} /\|u\|_{B}^{2}}-1=\frac{\lambda_{h, j}-\lambda_{k_{i}}}{\lambda_{k_{i}}}+\lambda_{h, j} \frac{D\left(\left(I-P_{h}\right) u, u_{h, j}-u\right)}{\left\|\left(I-P_{h}\right) u\right\|_{B}^{2}} .
$$

From (4.1) or the standard, well-known results for eigenvalue approximation we have

$$
\begin{aligned}
& \frac{\lambda_{h, j}-\lambda_{k_{i}}}{\lambda_{k_{i}}} \leq d_{i}\left(\sup _{\substack{u \in M\left(\lambda_{k_{i}}\right) \\
\|u\|_{B}=1}}\left\|\left(I-P_{h}\right) u\right\|_{B}\right)^{2} \\
& =d_{i}\left(\sqrt{\lambda_{k_{i}}} \sup _{\substack{u \in M\left(\lambda_{k_{i}}\right) \\
\|u\|_{D}=1}}\left\|\left(I-P_{h}\right) T u\right\|_{B}\right)^{2} \\
& \leq d_{i} \eta^{2}(h), \quad j=k_{i}, \ldots, k_{i}+q_{i}-1 \text {, }
\end{aligned}
$$

from (3.13a) we have

$$
\left\|\left(I-P_{h}\right) u\right\|_{D} \leq \eta(h)\left\|\left(I-P_{h}\right) u\right\|_{B},
$$

and from (3.26) and (3.31b) we have

$$
\begin{aligned}
\left\|u_{h, j}-u\right\|_{D} & =\left\|u_{h, j}-E\left(\lambda_{k_{i}}\right) u_{h, j}\right\|_{D} \\
& \leq d_{i} \eta(h)\left\|u_{h, j}-E\left(\lambda_{k_{i}}\right) u_{h, j}\right\|_{B}=d_{i} \eta(h)\left\|\left(I-P_{h}\right) u\right\|_{B} .
\end{aligned}
$$

Combining (4.4)-(4.7), we obtain

$$
\begin{aligned}
\left|\frac{\left(\lambda_{h, j}-\lambda_{k_{i}}\right) / \lambda_{k_{i}}}{\left\|\left(I-P_{h}\right) u\right\|_{B}^{2} /\|u\|_{B}^{2}}-1\right| & \leq d_{i} \eta^{2}(h)+\frac{\lambda_{h, j} \mid D\left(\left(I-P_{h}\right) u, u_{h, j}-u \mid\right.}{\left\|\left(I-P_{h}\right) u\right\|_{B}^{2}} \\
& \leq d_{i} \eta^{2}+\frac{\lambda_{h, j}\left\|\left(I-P_{h}\right) u\right\|_{D}\left\|u_{h, j}-u\right\|_{D}}{\left\|\left(I-P_{h}\right) u\right\|_{B}^{2}} \leq d_{i} \eta^{2}
\end{aligned}
$$

the desired result.

Remark 4.1. Formula (4.4) is due to Chatelin [7], [8] and is used by her to prove (4.1). Using eigenvector estimates in $\|\cdot\|_{B}((3.26))$, one can prove

$$
\left|\frac{\left(\lambda_{h, j}-\lambda_{k_{i}}\right) / \lambda_{k_{i}}}{\left\|\left(I-P_{h}\right) E\left(u_{k_{i}}\right) u_{h, j}\right\|_{B}^{2} /\left\|E\left(\lambda_{k_{i}}\right) u_{h, j}\right\|_{B}^{2}}-1\right| \leq d_{i} \nu(h) \text {. }
$$

Inequality (4.2), which was proved using eigenvector estimates in $\|\cdot\|_{D}((3.31 \mathrm{~b})$ together with (3.26)), is an improvement over this result since, as we saw in Subsection 3.(b), $\eta^{2}$ may be of higher order than $\nu$.

Theorem 4.1 relates the eigenvalue error $\left(\lambda_{h, j}-\lambda_{k_{i}}\right) / \lambda_{k_{i}}$ to $\left\|\left(I-P_{h}\right) u\right\|_{B}^{2}$, with $u=E\left(\lambda_{k_{i}}\right) u_{h, j}$. We now prove a result that relates the eigenvalue error to $\left\|\left(I-P_{h}\right) u\right\|_{B}^{2} /\|u\|_{B}^{2}$, where $u \in M\left(\lambda_{k_{i}}\right)$ and $E_{h}\left(\lambda_{k_{i}}\right) u=u_{h, j}$, i.e., $u=u_{j}^{h}$, as defined in Subsection 3.(d).

THEOREM 4.2. For $i=1,2, \ldots$ there is a constant $d_{i}$ such that

$$
\left|\frac{\left(\lambda_{h, j}-\lambda_{k_{i}}\right) / \lambda_{k_{i}}}{\left\|\left(I-P_{h}\right) u\right\|_{B}^{2} /\|u\|_{B}^{2}}-1\right| \leq d_{i} \eta^{2}(h), \quad j=k_{i}, \ldots, k_{i}+q_{i}-1,
$$

where $u \in M\left(\lambda_{k_{i}}\right)$ satisfies $E_{h}\left(\lambda_{k_{i}}\right) u=u_{h, j}$. 
Proof. With $u \in M\left(\lambda_{k_{i}}\right)$ satisfying $E_{h}\left(\lambda_{k_{i}}\right) u=u_{h, j}$, we have

$$
\begin{aligned}
\left(\mu_{k_{i}}-\mu_{h, j}\right) B\left(u, u_{h, j}\right) & =B\left(T u, u_{h, j}\right)-B\left(u, T_{h} u_{h, j}\right) \\
& =B\left(T\left(I-P_{h}\right) u, u\right)+B\left(T\left(I-P_{h}\right) u, u_{h, j}-u\right) \\
& =\mu_{k_{i}}\left\|\left(I-P_{h}\right) u\right\|_{B}^{2}+B\left(T\left(I-P_{h}\right) u, u_{h, j}-u\right),
\end{aligned}
$$

from which we get, as above,

$$
\frac{\left(\lambda_{h, j}-\lambda_{k_{i}}\right) / \lambda_{k_{i}}}{\left\|\left(I-P_{h}\right) u\right\|_{B}^{2} /\left\|E_{h}\left(\lambda_{k_{i}}\right) u\right\|_{B}^{2}}-1=\frac{\lambda_{h, j}-\lambda_{k_{i}}}{\lambda_{k_{i}}}+\lambda_{h, j} \frac{D\left(\left(I-P_{h}\right) u, u_{h, j}-u\right)}{\left\|\left(I-P_{h}\right) u\right\|_{B}^{2}}
$$

It follows from (3.13a) and (3.16b) that

$$
\left\|u_{h, j}-u\right\|_{D} \leq d_{i} \eta\left\|\left(I-P_{h}\right) u\right\|_{B}
$$

Combining (4.5), (4.6), (4.9), and (4.10), we obtain

$$
\left|\frac{\left(\lambda_{h, j}-\lambda_{k_{i}}\right) / \lambda_{k_{i}}}{\left\|\left(I-P_{h}\right) u\right\|_{B}^{2} /\left\|E_{h}\left(\lambda_{k_{i}}\right) u\right\|_{B}^{2}}-1\right| \leq d_{i} \eta^{2}(h)
$$

from which we get

$$
\left|\frac{\left(\lambda_{h, j}-\lambda_{k_{i}}\right) / \lambda_{k_{i}}}{\left\|\left(I-P_{h}\right) u\right\|_{B}^{2} /\|u\|_{B}^{2}}-\frac{\|u\|_{B}^{2}}{\left\|E_{h}\left(\lambda_{k_{i}}\right) u\right\|_{B}^{2}}\right| \leq d_{i} \eta^{2}(h) \frac{\|u\|_{B}^{2}}{\left\|E_{h}\left(\lambda_{k_{i}}\right) u\right\|_{B}^{2}} .
$$

Since $u=\left(u-E_{h}\left(\lambda_{k_{i}}\right) u\right)+E_{h}\left(\lambda_{k_{i}}\right) u$ is an orthogonal decomposition in $H_{B}$, we have

$$
\|u\|_{B}^{2}=\left\|u-E_{h}\left(\lambda_{k_{i}}\right) u\right\|_{B}^{2}+\left\|E_{h}\left(\lambda_{k_{i}}\right) u\right\|_{B}^{2}
$$

and hence

$$
\frac{\|u\|_{B}^{2}}{\left\|E_{h}\left(\lambda_{k_{i}}\right) u\right\|_{B}^{2}}=1+\frac{\left\|u-E_{h}\left(\lambda_{k_{i}}\right) u\right\|_{B}^{2}}{\left\|E_{h}\left(\lambda_{k_{i}}\right) u\right\|_{B}^{2}} .
$$

Using (3.16a) and (2.13) we see that

$$
\frac{\left\|u-E_{h}\left(\lambda_{k_{i}}\right) u\right\|_{B}^{2}}{\left\|E_{h}\left(\lambda_{k_{i}}\right) u\right\|_{B}^{2}} \leq C \varepsilon_{u}^{2}(h) \leq C \eta^{2}(h) .
$$

Combining (4.11), (4.12), and (4.13), we get the desired result.

(b) The Eigenvector Error. Let $i=1,2, \ldots$ and let $j=k_{i}, \ldots, k_{i}+q_{i}-1$ be fixed, and consider $\bar{u}_{h, j}$ and $E\left(\lambda_{k_{i}}\right) \bar{u}_{h, j}$ (recall that $\left\|\bar{u}_{h, j}\right\|_{D}=1$ ). We showed in Subsection 3.(d) (see (3.31a)) that

$$
\left\|E\left(\lambda_{k_{i}}\right) \bar{u}_{h, j}-\bar{u}_{h, j}\right\|_{D} \leq d_{i} \eta(h)\left\|E\left(\lambda_{k_{i}}\right) \bar{u}_{h, j}-\bar{u}_{h, j}\right\|_{B}
$$

From Lemma 3.1 we have

$$
\lambda_{h, j}-\lambda_{k_{i}}=\left\|E\left(\lambda_{k_{i}}\right) \bar{u}_{h, j}-\bar{u}_{h, j}\right\|_{B}^{2}-\lambda_{k_{i}}\left\|E\left(\lambda_{k_{i}}\right) \bar{u}_{h, j}-\bar{u}_{h, j}\right\|_{D}^{2} .
$$

Combining (4.14) and (4.15), we obtain

$$
\lambda_{h, j}-\lambda_{k_{i}} \geq\left\|E\left(\lambda_{k_{i}}\right) \bar{u}_{h, j}-\bar{u}_{h, j}\right\|_{B}^{2}\left(1-d_{i} \eta^{2}(h)\right),
$$

which implies

$$
\frac{\left\|E\left(\lambda_{k_{i}}\right) \bar{u}_{h, j}-\bar{u}_{h, j}\right\|_{B}^{2}}{\left\|\left(I-P_{h}\right) E\left(\lambda_{k_{i}}\right) \bar{u}_{h, j}\right\|_{B}^{2}} \leq \frac{\lambda_{h, j}-\lambda_{k_{i}}}{\left\|\left(I-P_{h}\right) E\left(\lambda_{k_{i}}\right) \bar{u}_{h, j}\right\|_{B}^{2}\left(1-d_{i} \eta^{2}\right)} .
$$


Since $\bar{u}_{h, j}=E\left(\lambda_{k_{i}}\right) \bar{u}_{h, j}+\left(\bar{u}_{h, j}-E\left(\lambda_{k_{i}}\right) \bar{u}_{h, j}\right)$ is an orthogonal decomposition in $H_{D}$, we have

$$
1=\left\|E\left(\lambda_{k_{i}}\right) \bar{u}_{h, j}\right\|_{D}^{2}+\left\|\bar{u}_{h, j}-E\left(\lambda_{k_{i}}\right) \bar{u}_{h, j}\right\|_{D}^{2} .
$$

From this, (3.26), and (4.14) we get

$$
\begin{aligned}
1 & \leq\left\|E\left(\lambda_{k_{i}}\right) \bar{u}_{h, j}\right\|_{D}^{2}+d_{i} \eta^{2}\left\|\left(P_{h}-I\right) E\left(\lambda_{k_{i}}\right) \bar{u}_{h, j}\right\|_{B}^{2} \\
& =\frac{\left\|E\left(\lambda_{k_{i}}\right) \bar{u}_{h, j}\right\|_{B}^{2}}{\lambda_{k_{i}}}\left(1+d_{i} \eta^{4}\right) .
\end{aligned}
$$

Now, combining (4.2), (4.16), and (4.17), we have

$$
\begin{aligned}
& \frac{\left\|E\left(\lambda_{k_{i}}\right) \bar{u}_{h, j}-\bar{u}_{h, j}\right\|_{B}}{\left\|\left(I-P_{h}\right) E\left(\lambda_{k_{i}}\right) \bar{u}_{h, j}\right\|_{B}} \\
& \quad \leq\left\{\frac{\left(\lambda_{h, j}-\lambda_{k_{i}}\right) / \lambda_{k_{i}}}{\left\|\left(I-P_{h}\right) E\left(\lambda_{k_{i}}\right) \bar{u}_{h, j}\right\|_{B}^{2} /\left\|E\left(\lambda_{k_{i}}\right) \bar{u}_{h, j}\right\|_{B}^{2}} \times \frac{1+d_{i} \eta^{4}}{1-d_{i} \eta^{2}}\right\}^{1 / 2} \\
& \quad \leq\left\{\left(1+d_{i} \eta^{2}\right) \frac{\left(1+d_{i} \eta^{4}\right)}{1-d_{i} \eta^{2}}\right\}^{1 / 2} \leq\left[1+d_{i} \eta^{2}\right]^{1 / 2} \leq 1+d_{i} \eta^{2}(h) .
\end{aligned}
$$

We summarize this (cf. (1.3b) and (1.4)) in

THEOREM 4.3. For $i=1,2, \ldots$ there is a constant $d_{i}$ such that

$$
1 \leq \frac{\left\|E\left(\lambda_{k_{i}}\right) \bar{u}_{h, j}-\bar{u}_{h, j}\right\|_{B}}{\left\|\left(I-P_{h}\right) E\left(\lambda_{k_{i}}\right) \bar{u}_{h, j}\right\|_{B}} \leq 1+d_{i} \eta^{2}(h), \quad j=k_{i}, \ldots, k_{i}+q_{i}-1 .
$$

In (4.18), $\bar{u}_{h, j}$ can be replaced by $u_{h, j}$.

Remark 4.2. The result (4.18) is stronger than (3.26) since $\eta^{2}$ may be of higher order than $\nu$.

Next consider $\bar{u}_{h, j}$ and $\bar{u}_{j}^{h}$ (recall that $\bar{u}_{j}^{h} \in M\left(\lambda_{k_{i}}\right)$ satisfies $E_{h}\left(\lambda_{k_{i}}\right) \bar{u}_{j}^{h}=\bar{u}_{h, j}$ ). We know (see (3.28a)) that

$$
\left\|\bar{u}_{j}^{h}-\bar{u}_{h, j}\right\|_{D} \leq d_{i} \eta(h)\left\|\bar{u}_{j}^{h}-\bar{u}_{h, j}\right\|_{B} .
$$

This, together with Lemma 3.1, yields

$$
\begin{aligned}
\lambda_{h, j}-\lambda_{k_{i}} & =\left\|\bar{u}_{j}^{h}-\bar{u}_{h, j}\right\|_{B}^{2}-\lambda_{k_{i}}\left\|\bar{u}_{j}^{h}-\bar{u}_{h, j}\right\|_{D}^{2} \\
& \geq\left\|\bar{u}_{j}^{h}-\bar{u}_{h, j}\right\|_{B}^{2}\left(1-d_{i} \eta^{2}\right),
\end{aligned}
$$

which implies

$$
\begin{aligned}
\frac{\left\|\bar{u}_{j}^{h}-\bar{u}_{h, j}\right\|_{B}^{2}}{\left\|\left(I-P_{h}\right) \bar{u}_{j}^{h}\right\|_{B}^{2}} & \leq \frac{\lambda_{h, j}-\lambda_{k_{i}}}{\left\|\left(I-P_{h}\right) \bar{u}_{j}^{h}\right\|_{B}^{2}\left(1-d_{i} \eta^{2}\right)} \\
& =\frac{\left(\lambda_{h, j}-\lambda_{k_{i}}\right) / \lambda_{k_{i}}}{\left\|\left(I-P_{h}\right) \bar{u}_{j}^{h}\right\|_{B}^{2}\left(1-d_{i}^{2} \eta\right) /\left\|\bar{u}_{j}^{h}\right\|_{B}^{2}} .
\end{aligned}
$$

Finally, combining (4.8) and (4.19), we have

$$
1 \leq \frac{\left\|\bar{u}_{j}^{h}-\bar{u}_{h, j}\right\|_{B}}{\left\|\left(I-P_{h}\right) \bar{u}_{j}^{h}\right\|_{B}} \leq\left(\frac{1+d_{i} \eta^{2}}{1-d_{i} \eta^{2}}\right)^{1 / 2} \leq 1+d_{i} \eta^{2} .
$$

This result (cf. (1.3a) and (1.4)) and the related result (3.19) are summarized in 
THEOREM 4.4. For $i=1,2, \ldots$ there is a constant $d_{i}$ such that

$$
\begin{aligned}
& 1 \leq \frac{\left\|\bar{u}_{j}^{h}-\bar{u}_{h, j}\right\|_{B}}{\left\|\left(I-P_{h}\right) \bar{u}_{j}^{h}\right\|_{B}}=\frac{\left\|\bar{u}_{j}^{h}-E_{h}\left(\lambda_{k_{i}}\right) \bar{u}_{j}^{h}\right\|_{B}}{\left\|\left(I-P_{h}\right) \bar{u}_{j}\right\|_{B}} \leq 1+d_{i} \eta^{2}(h), \\
& j=k_{i}, \ldots, k_{i}+q_{i}-1 .
\end{aligned}
$$

The inequalities (4.20) remain valid if $\bar{u}_{h, j}$ is replaced by $u_{h, j}$ and $\bar{u}_{j}^{h}$ by $u_{j}^{h}$. There is a constant $d_{i}$ such that

$$
1 \leq \frac{\left\|u-E_{h}\left(\lambda_{k_{i}}\right) u\right\|_{B}}{\left\|\left(u-P_{h}\right) u\right\|_{B}} \leq 1+d_{i} \nu(h) \quad \text { for all } u \in M\left(\lambda_{k_{i}}\right) .
$$

Remark 4.3. We have restated (3.19) in (4.21) because it is related to (4.20) and it is the strongest known result of its specific type. It should be noted that (4.21) is true for all $u \in M\left(\lambda_{k_{i}}\right)$, whereas (4.20) is valid only for $u=\bar{u}_{j}^{h}, j=k_{i}, \ldots, k_{i}+q_{i}-1$. However, for these $u$ 's, (4.20) is stronger than (4.21).

Remark 4.4. The eigenvector estimates (4.18) and (4.20) were obtained from the eigenvalue estimates (4.2) and (4.8), respectively, via Lemma 3.1, which provides an estimate for the eigenvector error in terms of the eigenvalue error. Estimates for eigenvector error in terms of eigenvalue error can be found in Weinberger [13].

Remark 4.5. See [2], [4] for a numerical study of the reliability of the results of this section-which are of an asymptotic nature - as a guide to practical computations, which often take place in the preasymptotic phase.

5. An Additional Result for Multiple Eigenvalues. Estimate (1.3c) implies that

$$
\lambda_{h, k_{i}}-\lambda_{k_{i}} \leq C \frac{\inf _{\chi \in S_{h}}\left\|E\left(\lambda_{k_{i}}\right) u_{h, k_{i}}-\chi\right\|_{B}^{2}}{\left\|E\left(\lambda_{k_{i}}\right) u_{h, k}\right\|_{B}^{2}}
$$

and estimate (4.8) shows that

$$
\lambda_{h, k_{i}}-\lambda_{k_{i}} \leq C \inf _{\chi \in S_{h}}\|u-\chi\|_{B}^{2} /\|u\|_{B}^{2}
$$

where $u \in M\left(\lambda_{k_{i}}\right)$ satisfies $E_{h}\left(\lambda_{k_{i}}\right)=u_{h, k_{i}}$. In [3], Babuška and Osborn proved the stronger result (cf. (1.5))

$$
\lambda_{h, k_{i}}-\lambda_{k_{i}} \leq C \inf _{\substack{u \in M\left(\lambda_{k_{i}}\right) \\\|u\|_{B}=1}} \inf _{\chi \in S_{h}}\|u-\chi\|_{B}^{2}
$$

(as well as similar estimates for $\lambda_{h, j}-\lambda_{k_{i}}, j=k_{i}+1, \ldots, k_{i}+q_{i}-1$, and for the eigenvector errors), which shows that $\lambda_{h, k_{i}}-\lambda_{k_{i}}$, the error in the approximate eigenvalue closest to $\lambda_{k_{i}}$, is governed by the approximability of the exact eigenvector corresponding to $\lambda_{k_{i}}$ that can be best approximated by $S_{h}$. In this section we give a simplified proof of the results of [3], which in addition provides information on $C$ (the results in [3] only established that $C$ is a constant), and we estimate the eigenvector error in $\|\cdot\|_{D}$ and $\|\cdot\|_{-B}$.

As above, for $i=1,2, \ldots$ suppose $k_{i}$ is the lowest index of the $i$ th distinct eigenvalue of (2.5) and let $q_{i}$ be its multiplicity, i.e., suppose

$$
\lambda_{k_{i-1}+q_{i-1}-1}=\lambda_{k_{i}-1}<\lambda_{k_{i}}=\lambda_{k_{i}+1}=\cdots=\lambda_{k_{i}+q_{i}-1}<\lambda_{k_{i}+q_{i}}=\lambda_{k_{i+1}} .
$$


Let

$$
\varepsilon_{i, j}(h)=\inf _{\substack{u \in M\left(\lambda_{k_{i}}\right) \\\|u\|_{B}=1}} \inf _{\chi \in S_{h}}\|u-\chi\|_{B}
$$

where $M\left(\lambda_{k_{i}}\right)$ is defined in (2.9). The restrictions

$$
B\left(u, u_{h, k_{i}}\right)=\cdots=B\left(u, u_{h, k_{i}+j-2}\right)=0
$$

are considered vacuous if $j=1$. We note that they are equivalent to

$$
B\left(u, E\left(\lambda_{k_{i}}\right) u_{h, l}\right)=0, \quad l=k_{i}, \ldots, k_{i}+j-2,
$$

and to

$$
B\left(E_{h}\left(\lambda_{k_{i}}\right) u, u_{h, l}\right)=0, \quad l=k_{i}, \ldots, k_{i}+j-2 .
$$

THEOREM 5.1 (cf. (1.5)). For $i=1,2, \ldots$ there is a function $\bar{C}_{i}(h)$ and a constant $C_{i}$, with

$$
\bar{C}_{i}(h) \leq 1+d_{i} \nu(h), \quad d_{i}=\text { constant }
$$

such that

$$
\left(\lambda_{h, k_{i}+j-1}-\lambda_{k_{i}}\right) / \lambda_{k_{i}} \leq \bar{C}_{i}(h) \varepsilon_{i, j}^{2}(h), \quad j=1, \ldots, q_{i}
$$

and such that the eigenvectors $u_{1}, u_{2}, \ldots$ of (2.5) can be chosen so that (2.6) is satisfied and such that

$$
\begin{aligned}
\left\|u_{h, k_{i}+j-1}-u_{k_{i}+j-1}\right\|_{B} & \leq \bar{C}_{i}(h) \varepsilon_{i, j}(h), \quad j=1, \ldots, q_{i}, \\
\left\|u_{h, k_{i}+j-1}-u_{k_{i}+j-1}\right\|_{D} & \leq C_{i} \eta(h) \varepsilon_{i, j}(h), \quad j=1, \ldots, q_{i},
\end{aligned}
$$

and

$$
\left\|u_{h, k_{i}+j-1}-u_{k_{i}+j-1}\right\|_{-B} \leq C_{i} \nu(h) \varepsilon_{i, j}(h), \quad j=1, \ldots, q_{i},
$$

where $\eta(h)$ and $\nu(h)$ are defined in (3.7) and (3.8).

Proof. Let $i$ and $j$, with $i=1,2, \ldots$ and $j=1, \ldots, q_{i}$, be fixed. Note that $\varepsilon_{u}(h) \leq \lambda_{k_{i}} \nu(h)$ for all $u \in M\left(\lambda_{k_{i}}\right)$ and $\varepsilon_{i, j}(h) \leq \lambda_{k_{i}} \nu(h), j=1, \ldots, q_{i}$. Let $u \in M\left(\lambda_{k_{i}}\right)$ with $B\left(u, u_{h, k_{i}}\right)=\cdots=B\left(u, u_{h, k_{i}+j-2}\right)=0$ and $\|u\|_{B}=1$. Now apply $\left(2.7^{h}\right)$ and Lemma 3.1 with $(\lambda, u)=\left(\lambda_{k_{i}}, u /\left\|E_{h}\left(\lambda_{k_{i}}\right) u\right\|_{D}\right)$ and $w=$ $E_{h}\left(\lambda_{k_{i}}\right) u /\left\|E_{h}\left(\lambda_{k_{i}}\right) u\right\|_{D}$. Since

$$
B\left(E_{h}\left(\lambda_{k_{i}}\right) u, u_{h, l}\right)=0, \quad l=1, \ldots, k_{i}-1,
$$

by the orthogonality of the approximate eigenvectors, and

$$
B\left(E_{h}\left(\lambda_{k_{i}}\right) u, u_{h, l}\right)=B\left(u, u_{h, l}\right)=0, \quad l=k_{i}, \ldots, k_{i}+j-2,
$$

by the assumption on $u$, we have

$$
\begin{aligned}
\lambda_{h, k_{i}+j-1}-\lambda_{k_{i}} & \leq B\left(\frac{E_{h}\left(\lambda_{k_{i}}\right) u}{\left\|E_{h}\left(\lambda_{k_{i}}\right) u\right\|_{D}}, \frac{E_{h}\left(\lambda_{k_{i}}\right) u}{\left\|E_{h}\left(\lambda_{k_{i}}\right) u\right\|_{D}}\right)-\lambda_{k_{i}} \\
& =\frac{\left\|E_{h}\left(\lambda_{k_{i}}\right) u-u\right\|_{B}^{2}-\lambda_{k_{i}}\left\|E_{h}\left(\lambda_{k_{i}}\right) u-u\right\|_{D}^{2}}{\left\|E_{h}\left(\lambda_{k_{i}}\right) u\right\|_{D}^{2}} \\
& \leq \frac{\left\|E_{h}\left(\lambda_{k_{i}}\right) u-u\right\|_{B}^{2}}{\left\|E_{h}\left(\lambda_{k_{i}}\right) u\right\|_{D}^{2}}
\end{aligned}
$$


From (3.19) we have

$$
\left\|E_{h}\left(\lambda_{k_{i}}\right) u-u\right\|_{B} \leq(1+d \nu)\left\|u-P_{h} u\right\|_{B} .
$$

From (3.13) and (3.16b) we see that

$$
\begin{aligned}
\left|\left\|E_{h}\left(\lambda_{k_{i}}\right) u\right\|_{D}-\lambda_{k_{i}}^{-1 / 2}\right| & =\left|\left\|E_{h}\left(\lambda_{k_{i}}\right) u\right\|_{D}-\|u\|_{D}\right| \\
& \leq\left\|E_{h}\left(\lambda_{k_{i}}\right) u-u\right\|_{D} \leq d\left\|u-P_{h} u\right\|_{D} \\
& \leq d \eta\left\|u-P_{h} u\right\|_{B}=d \eta(h) \varepsilon_{u}(h),
\end{aligned}
$$

which shows that

$$
\begin{aligned}
\frac{1}{\lambda_{k_{i}}\left\|E_{h}\left(\lambda_{k_{i}}\right) u\right\|_{D}^{2}} & \leq\left[1+\frac{d \eta \varepsilon_{u}(h)}{\left\|E_{h}\left(\lambda_{k_{i}}\right) u\right\|_{D}}\right]^{2} \\
& \leq 1+d \eta \varepsilon_{u}(h) \leq 1+d \eta \nu .
\end{aligned}
$$

Combining (5.6)-(5.8), we get

$$
\begin{aligned}
\left(\lambda_{h, k_{i}+j-1}-\lambda_{k_{i}}\right) / \lambda_{k_{i}} & \leq(1+d \nu)^{2}(1+d \eta \nu)\left\|u-P_{h} u\right\|_{B}^{2} \\
& \leq(1+d \nu)\left\|u-P_{h} u\right\|_{B}^{2} .
\end{aligned}
$$

Now since (5.9) holds for all $u \in M\left(\lambda_{k_{i}}\right)$ with $B\left(u, u_{h, l}\right)=0, l=k_{i}, \ldots, k_{i}+j-2$, and $\|u\|_{B}=1$, we have

$$
\begin{aligned}
& \left(\lambda_{h, k_{i}+j-1}-\lambda_{k_{i}}\right) / \lambda_{k_{i}} \\
& \leq(1+d \nu(h))\left(\inf _{\substack{u \in M\left(\lambda_{k_{i}}\right) \\
\|u\|_{B}=1}} \inf _{\chi \in S_{h}}\|u-\chi\|_{B}\right)^{2} \\
& =\left(1+d_{i} \nu(h)\right) \varepsilon_{i, j}^{2}(h),
\end{aligned}
$$

which is (5.3) with $\bar{C}_{i}(h)=1+d_{i} \nu(h)$. Thus (5.2) and (5.3) have both been proved.

Remark 5.1. The minimum principle $\left(2.7^{h}\right)$ and Lemma 3.1 lead to a particularly simple proof of a result slightly weaker than (5.3) for the case $i=j=1$. It follows immediately from these two results that

$$
\begin{aligned}
\lambda_{h, 1}-\lambda_{1} & \leq B\left(\frac{P_{h} u}{\left\|P_{h} u\right\|_{D}}, \frac{P_{h} u}{\left\|P_{h} u\right\|_{D}}\right)-\lambda_{1} \\
& =\frac{\left\|P_{h} u-u\right\|_{B}^{2}-\lambda_{1}\left\|P_{h} u-u\right\|_{D}^{2}}{\left\|P_{h} u\right\|_{D}^{2}} \leq \frac{\left\|P_{h} u-u\right\|_{B}^{2}}{\left\|P_{h} u\right\|_{D}^{2}} \quad \forall u \in M\left(\lambda_{1}\right),
\end{aligned}
$$

and hence

$$
\left(\lambda_{h, 1}-\lambda_{1}\right) / \lambda_{1} \leq \inf _{\substack{u \in M\left(\lambda_{1}\right) \\\|u\|_{B}=1}} \frac{\left\|P_{h} u-u\right\|_{B}^{2}}{\lambda_{1}\left\|P_{h} u\right\|_{D}^{2}} \leq \bar{C}_{i}(h) \varepsilon_{1,1}(h),
$$

where $\bar{C}_{i}(h) \rightarrow 1$.

Now consider (5.4) and (5.5). Let $i=1,2, \ldots$ and $j=1, \ldots, q_{i}$ be fixed. Let $u_{h, k_{i}+j-1}^{\prime} \in M\left(\lambda_{k_{i}}\right)$ satisfy $E_{h}\left(\lambda_{k_{i}}\right) u_{k_{i}+j-1}^{\prime}=u_{h, k_{i}+j-1}\left(u_{k_{i}+j-1}^{\prime}=u_{k_{i}+j-1}^{h}\right.$, where $u_{k_{i}+j-1}^{h}$ was introduced in Subsection 3.(d)). Applying Lemma 3.1 with

$$
(\lambda, u)=\left(\lambda_{k_{i}}, \frac{u_{k_{i}+j-1}^{\prime}}{\left\|u_{h, k_{i}+j-1}\right\|_{D}}\right) \quad \text { and } \quad w=\frac{u_{h, k_{i}+j-1}}{\left\|u_{h, k_{i}+j-1}\right\|_{D}},
$$


we get

$$
\begin{aligned}
\lambda_{h, k_{i}+j-1}-\lambda_{k_{i}}= & \left\|\frac{u_{h, k_{i}+j-1}}{\left\|u_{h, k_{i}+j-1}\right\|_{D}}-\frac{u_{k_{i}+j-1}^{\prime}}{\left\|u_{h, k_{i}+j-1}\right\|_{D}}\right\|_{B}^{2} \\
& -\lambda_{k_{i}}\left\|\frac{u_{h, k_{i}+j-1}}{\left\|u_{h, k_{i}+j-1}\right\|_{D}}-\frac{u_{k_{i}+j-1}^{\prime}}{\left\|u_{h, k_{i}+j-1}\right\|_{D}}\right\|_{D}^{2}
\end{aligned}
$$

From (3.28b) we have

$$
\left\|u_{k_{i}+j-1}^{\prime}-u_{h, k_{i}+j-1}\right\|_{D} \leq C \eta(h)\left\|u_{k_{i}+j-1}^{\prime}-u_{h, k_{i}+j-1}\right\|_{B} .
$$

(5.10) and (5.11) yield

$$
\lambda_{h, k_{i}+j-1}-\lambda_{k_{i}} \geq \frac{\left[1-\lambda_{k_{i}} C^{2} \eta^{2}(h)\right]}{\left\|u_{h, k_{i}+j-1}\right\|_{D}^{2}}\left\|u_{h, k_{i}+j-1}-u_{k_{i}+j-1}^{\prime}\right\|_{B}^{2},
$$

which, together with (2.11), (2.12), and (5.3), yields

$$
\begin{aligned}
\left\|u_{k_{i}+j-1}^{\prime}-u_{h, k_{i}+j-1}\right\|_{B} & \leq \frac{\left(\lambda_{h, k_{i}+j-1}-\lambda_{k_{i}}\right)^{1 / 2}}{\lambda_{h, k_{i}+j-1}^{1 / 2}\left[1-\lambda_{k_{i}} C^{2} \eta^{2}\right]^{1 / 2}} \\
& \leq \frac{\lambda_{k_{i}}^{1 / 2} \bar{C}_{i}(h)^{1 / 2} \varepsilon_{i, j}(h)}{\lambda_{h, k_{i}+j-1}^{1 / 2}\left[1-\lambda_{k_{i}} C^{2} \eta^{2}\right]^{1 / 2}} \\
& \leq \bar{C}_{i}(h) \varepsilon_{i, j}(h), \quad j=1, \ldots, q_{i},
\end{aligned}
$$

where, because of $(5.2), \bar{C}_{i}(h) \leq 1+d_{i} \nu(h)$. (5.12) shows that the $u_{k_{i}+j-1}^{\prime}$ satisfy estimates (5.4). The inequality (5.12), together with $(3.28 \mathrm{~b})$ and $(3.29 \mathrm{~b})$, shows that the $u_{k_{i}+j-1}^{\prime}$ satisfy estimates (5.5). They will not in general, however, be orthonormal with respect to $B$, so that (2.6) may not be satisfied.

It remains to modify the $u_{k_{i}+j-1}^{\prime}$, i.e., replace $u_{k_{i}+j-1}^{\prime}$ by $u_{k_{i}+j-1}$, in such a way that (2.6) and (5.4) and (5.5) hold. We proceed by induction on $j$. Let $j=1$. If we define $u_{k_{i}}=u_{k_{i}}^{\prime} /\left\|u_{k_{i}}^{\prime}\right\|_{B}$, we have $\left\|u_{k_{i}}\right\|_{B}=1$, so that (2.6) is satisfied. From (5.12) we have

$$
\begin{aligned}
\left|\left\|u_{k_{i}+j-1}^{\prime}\right\|_{B}-1\right| & =\left|\left[1+\left\|u_{k_{i}+j-1}^{\prime}-u_{h, k_{i}+j-1}\right\|_{B}^{2}\right]^{1 / 2}-1\right| \\
& \leq \frac{\left\|u_{k_{i}+j-1}^{\prime}-u_{h, k_{i}+j-1}\right\|_{B}^{2}}{2} \leq C \nu \varepsilon_{i, j}, \quad j=1, \ldots, q_{i},
\end{aligned}
$$

and hence

$$
\begin{aligned}
\left\|u_{k_{i}}-u_{h, k_{i}}\right\|_{B} & \leq\left\|u_{k_{i}}-u_{k_{i}}^{\prime}\right\|_{B}+\left\|u_{k_{i}}^{\prime}-u_{h, k_{i}}\right\|_{B} \\
& \leq\left|\left\|u_{k_{i}}^{\prime}\right\|_{B}-1\right|+\left\|u_{k_{i}}^{\prime}-u_{h, k_{i}}\right\|_{B} \\
& \leq C \nu \varepsilon_{i, 1}+\bar{C}_{i}(h) \eta \varepsilon_{i, 1} \leq \bar{C}_{i}(h) \varepsilon_{i, 1}(h)
\end{aligned}
$$

where $\bar{C}_{i}(h) \leq 1+d_{i} \nu(h)$, which is (5.4) for $j=1$. Using (3.9), (5.13), and the fact that the $u_{k_{i}+j-1}^{\prime}$ satisfy $(5.5 \mathrm{a})$, we get

$$
\begin{aligned}
\left\|u_{k_{i}}-u_{h, k_{i}}\right\|_{D} & \leq\left\|u_{k_{i}}-u_{k_{i}}^{\prime}\right\|_{D}+\left\|u_{k_{i}}^{\prime}-u_{h, k_{i}}\right\|_{D} \\
& =\lambda_{k_{i}}^{-1 / 2}\left|\left\|u_{k_{i}}^{\prime}\right\|_{B}-1\right|+\left\|u_{k_{i}}^{\prime}-u_{h, k_{i}}\right\|_{D} \\
& \leq C \nu \varepsilon_{i, 1}+C \eta \varepsilon_{i, 1} \leq C_{i} \eta(h) \varepsilon_{i, 1}(h),
\end{aligned}
$$

which is (5.5a) for $j=1$. A similar estimate establishes (5.5b) for $j=1$. 
Next suppose $j=2$. Let $u_{k_{i}}^{\prime \prime}=u_{k_{i}+1}^{\prime}-B\left(u_{k_{i}+1}^{\prime}, u_{k_{i}}\right) u_{k_{i}}$. Using (5.4) for $j=1$, (5.5b) for $j=1,(5.12)$, and the facts that (5.5b) holds for the $u_{k_{i}+j-1}^{\prime}$ and that $\varepsilon_{i, 1} \leq \varepsilon_{i, 2}$, we have

$$
\begin{aligned}
\left|B\left(u_{k_{i}+1}^{\prime}, u_{k_{i}}\right)\right| \leq & \left|B\left(u_{k_{i}+1}^{\prime}-u_{h, k_{i}+1}, u_{k_{i}}\right)\right| \\
& +\left|B\left(u_{h, k_{i}+1}-u_{k_{i}+1}^{\prime}, u_{k_{i}}-u_{h, k_{i}}\right)\right| \\
& +\left|B\left(u_{k_{i}+1}^{\prime}, u_{k_{i}}-u_{h, k_{i}}\right)\right| \\
= & \lambda_{k_{i}}\left|D\left(u_{k_{i}+1}^{\prime}-u_{h, k_{i}+1}, u_{k_{i}}\right)\right| \\
& +\left|B\left(u_{h, k_{i}+1}-u_{k_{i}+1}^{\prime}, u_{k_{i}}-u_{h, k_{i}}\right)\right| \\
& +\lambda_{k_{i}+1}\left|D\left(u_{k_{i}+1}^{\prime}, u_{k_{i}}-u_{h, k_{i}}\right)\right| \\
\leq & \lambda_{k_{i}}\left\|u_{k_{i}+1}^{\prime}-u_{h, k_{i}+1}\right\|_{-B}\left\|u_{k_{i}}\right\|_{B} \\
& +\left\|u_{h, k_{i}+1}-u_{k_{i}+1}^{\prime}\right\|_{B}\left\|u_{k_{i}}-u_{h, k_{i}}\right\|_{B} \\
& +\lambda_{k_{i}+1}\left\|u_{k_{i}+1}^{\prime}\right\|_{B}\left\|u_{k_{i}}-u_{h, k_{i}}\right\|_{-B} \\
\leq & \lambda_{k_{i}}\left\|u_{k_{i}+1}^{\prime}-u_{h, k_{i}+1}\right\|_{-B} \\
& +\left\|u_{h, k_{i}+1}-u_{k_{i}+1}^{\prime}\right\|_{B}\left\|u_{k_{i}}-u_{h, k_{i}}\right\|_{B} \\
& +\lambda_{k_{i}}\left(1+\left\|u_{k_{i}+1}^{\prime}-u_{h, k_{i}+1}\right\|_{B}\right)\left\|u_{k_{i}}-u_{h, k_{i}}\right\|_{-B} \\
\leq & C \nu \varepsilon_{i, 2}+\bar{C}_{i}(h) \varepsilon_{i, 2} \bar{C}_{i}(h) \varepsilon_{i, 1}+C \nu \bar{C}_{i}(h) \varepsilon_{i, 1} \\
\leq & C \nu(h) \varepsilon_{i, 2}(h),
\end{aligned}
$$

and hence

$$
\left\|u_{k_{i}+1}^{\prime}-u_{k_{i}+1}^{\prime \prime}\right\|_{B}=\left|B\left(u_{k_{i}+1}^{\prime}, u_{k_{i}}\right)\right| \leq C \nu(h) \varepsilon_{i, 2}(h) .
$$

Now set $u_{k_{i}+1}=u_{k_{i}+1}^{\prime \prime} /\left\|u_{k_{i}+1}^{\prime \prime}\right\|_{B}$. Combining (5.12), (5.13), and (5.15), we obtain

$$
\begin{aligned}
\left\|u_{k_{i}+1}-u_{h, k_{i}+1}\right\|_{B} \leq & \left\|u_{k_{i}+1}-u_{k_{i}+1}^{\prime \prime}\right\|_{B}+\left\|u_{k_{i}+1}^{\prime \prime}-u_{h, k_{i}+1}\right\|_{B} \\
= & \left|\left\|u_{k_{i}+1}^{\prime \prime}\right\|_{B}-1\right|+\left\|u_{k_{i}+1}^{\prime \prime}-u_{h, k_{i}+1}\right\|_{B} \\
\leq & \left|\left\|u_{k_{i}+1}^{\prime}\right\|_{B}-1\right|+2\left\|u_{k_{i}+1}^{\prime \prime}-u_{k_{i}+1}^{\prime}\right\|_{B}+\left\|u_{k_{i}+1}^{\prime}-u_{h, k_{i}+1}\right\|_{B} \\
& +C \nu \varepsilon_{i, 2}(h)+C \nu \varepsilon_{i, 2}(h)+\bar{C}_{i}(h) \varepsilon_{i, 2}(h) \\
\leq & \bar{C}_{i}(h) \varepsilon_{i, 2}(h),
\end{aligned}
$$

where $\bar{C}_{i}(h) \leq 1+d_{i} \nu(h)$, which is (5.4) for $j=2$.

Now consider (5.5a) for $j=2$. Using (5.13), (5.14), (5.15) and the fact that the $u_{k_{i}+j-1}^{\prime}$ satisfy (5.5), we have

$$
\begin{aligned}
\left\|u_{k_{i}+1}-u_{h, k_{i}+1}\right\|_{D} \leq & \left\|u_{k_{i}+1}-u_{k_{i}+1}^{\prime \prime}\right\|_{D}+\left\|u_{k_{i}+1}^{\prime \prime}-u_{h, k_{i}+1}\right\|_{D} \\
= & \lambda_{k_{i}}^{-1 / 2}\left|\left\|u_{k_{i}+1}^{\prime \prime}\right\|_{B}-1\right|+\left\|u_{k_{i}+1}^{\prime \prime}-u_{h, k_{i}+1}\right\|_{D} \\
\leq & \lambda_{k_{i}}^{-1 / 2}\left|\left\|u_{k_{i}+1}^{\prime}\right\|_{B}-1\right|+\lambda_{k_{i}}^{-1 / 2}\left\|u_{k_{i}+1}^{\prime \prime}-u_{k_{i}+1}^{\prime}\right\|_{B} \\
& +\left\|u_{k_{i}+1}^{\prime}-u_{h, k_{i}+1}\right\|_{D}+\left|B\left(u_{k_{i}+1}^{\prime}, u_{k_{i}}\right)\right|\left\|u_{k_{i}}\right\|_{D} \\
& +C \nu \varepsilon_{i, 2}+C \nu \varepsilon_{i, 2}+C \eta \varepsilon_{i, 2}+C \nu \varepsilon_{i, 2} \\
\leq & C_{i} \eta \varepsilon_{i, 2}(h)
\end{aligned}
$$

which is $(5.5 \mathrm{a})$ for $j=2$. The proof of $(5.5 \mathrm{~b})$ is similar.

Continuing in this manner we get $(2.6),(5.4)$, and (5.5) for $j=1, \ldots, q_{i}$. This completes the proof. 
THEOREM 5.2. For $i=1,2, \ldots$ there is a function $\hat{C}_{i}(h)$ with

$$
\hat{C}_{i}(h) \geq 1-d_{i} \nu(h), \quad d_{i} \geq 0 \text { constant }
$$

such that

$$
\left(\lambda_{h, k_{i}+j-1}-\lambda_{k_{i}}\right) / \lambda_{k_{i}} \geq \hat{C}_{i}(h) \varepsilon_{i, j}^{2}(h), \quad j=1, \ldots, q_{i}
$$

and

$$
\left\|u_{h, k_{i}+j-1}-u_{k_{i}+j-1}\right\|_{B} \geq \hat{C}_{i}(h) \varepsilon_{i, j}(h), \quad j=1, \ldots, q_{i} .
$$

Proof. First consider (5.18) for $j=1$. It is immediate that

$$
\left\|u_{h, k_{i}}-u_{k_{i}}\right\|_{B} \geq \inf _{\substack{u \in M\left(\lambda_{k_{i}}\right) \\\|u\|_{B}=1}} \inf _{\chi \in S_{h}}\|u-\chi\|_{B}=\varepsilon_{i, 1}(h) .
$$

Thus for $j=1$, (5.18) holds with $\hat{C}_{i}(h)=1$.

Now suppose $j=2$. Since

$$
\begin{aligned}
B\left(u_{k_{i}+1}^{\prime}, u_{h, k_{i}}\right) & =B\left(u_{k_{i}+1}^{\prime}, E_{h}\left(\lambda_{k_{i}}\right) u_{h, k_{i}}\right) \\
& =B\left(E_{h}\left(\lambda_{k_{i}}\right) u_{k_{i}+1}^{\prime}, u_{h, k_{i}}\right)=B\left(u_{h, k_{i}+1}, u_{h, k_{i}}\right)=0
\end{aligned}
$$

we see that

$$
\left\|u_{h, k_{i}+1}-u_{k_{i}+1}^{\prime}\right\|_{B} \geq \inf _{\substack{u \in M\left(\lambda_{k_{i}}\right) \\\|u\|_{B}=1 \\ B\left(u, u_{h, k_{i}}\right)=0}} \inf _{\chi \in S_{h}}\|u-\chi\|_{B}=\varepsilon_{i, 2}(h) .
$$

Combining this result with (5.13) and (5.15), we get

$$
\begin{aligned}
& \left\|u_{h, k_{i}+1}-u_{k_{i}+1}\right\|_{B} \\
& \quad \geq\left\|u_{h, k_{i}+1}-u_{k_{i}+1}^{\prime}\right\|_{B}-\left\|u_{k_{i}+1}^{\prime}-u_{k_{i}+1}^{\prime \prime}\right\|_{B}-\left\|u_{k_{i}+1}^{\prime \prime}-u_{k_{i}+1}\right\|_{B} \\
& \quad \geq\left\|u_{h, k_{i}+1}-u_{k_{i}+1}^{\prime}\right\|_{B}-2\left\|u_{k_{i}+1}^{\prime}-u_{k_{i}+1}^{\prime \prime}\right\|_{B}-\left|\left\|u_{k_{i}+1}^{\prime}\right\|_{B}-1\right| \\
& \quad \geq\left(1-d_{i} \nu\right) \varepsilon_{i, 2}(h)
\end{aligned}
$$

which is $(5.18)$ for $j=2$. Continuing in this manner, we get $(5.18)$ for $j=1, \ldots, q_{i}$.

Now consider (5.17). From Lemma 3.1, (5.5a), and (5.18) we see that

$$
\begin{aligned}
\left(\lambda_{h, k_{i}+j-1}-\lambda_{k_{i}}\right) / \lambda_{k_{i}} & =\frac{\left\|u_{h, k_{i}+j-1}-u_{k_{i}+j-1}\right\|_{B}^{2}}{\lambda_{k_{i}}\left\|u_{h, k_{i}+j-1}\right\|_{D}^{2}} \\
& -\frac{\left\|u_{h, k_{i}+j-1}-u_{k_{i}+j-1}\right\|_{D}^{2}}{\left\|u_{h, k_{i}+j-1}\right\|_{D}^{2}} \\
& \geq \frac{\lambda_{h, k_{i}+j-1}}{\lambda_{k_{i}}}\left(\left(1-d_{i} \nu\right)^{2}-\lambda_{k_{i}} C_{i}^{2} \eta^{2}\right) \varepsilon_{i, j}^{2}
\end{aligned}
$$

which implies (5.17).

Remark 5.2. Note that in Theorems 5.1 and 5.2 we have shown that

$$
\left|\frac{\left(\lambda_{h, k_{i}+j-1}-\lambda_{k_{i}}\right) / \lambda_{k_{i}}}{\varepsilon_{i, j}^{2}(h)}-1\right| \leq d_{i} \nu(h)
$$

whereas in Theorems 4.1 and 4.2 we showed that

$$
\left|\frac{\left(\lambda_{h, j}-\lambda_{k_{i}}\right) / \lambda_{k_{i}}}{\left\|\left(I-P_{h}\right) E\left(\lambda_{k_{i}}\right) u_{h, j}\right\|_{B}^{2} /\left\|E\left(\lambda_{k_{i}}\right) u_{h, j}\right\|_{B}^{2}}-1\right| \leq d_{i} \eta^{2}(h)
$$


and

$$
\left|\frac{\left(\lambda_{h, j}-\lambda_{k_{i}}\right) / \lambda_{k_{i}}}{\left\|\left(I-P_{h}\right) u\right\|_{B}^{2} /\|u\|_{B}^{2}}-1\right| \leq d_{i} \eta^{2}(h),
$$

for $u \in M\left(\lambda_{k_{i}}\right)$ with $E_{h}\left(\lambda_{k_{i}}\right) u=u_{h, j}$.

Remark 5.3. For a computational illustration of the results in this section see $[3],[4]$.

Institute for Physical Science and Technology

and Department of Mathematics

University of Maryland

College Park, Maryland 20742

Department of Mathematics

University of Maryland

College Park, Maryland 20742

E-mail: jeo@julia.umd.edu

1. J. P. AUBIN, "Behavior of the error of the approximate solution of boundary value problems for linear elliptic operators by Galerkin's and finite difference methods," Ann. Scuola Norm. Sup. Pisa, v. 21, 1967, pp. 599-637.

2. I. BABUSKA, B. Q. GUO \& J. E. OSBORN, "Regularity and numerical solution of eigenvalue problems with piecewise analytic data," SIAM J. Numer. Anal. (To appear.)

3. I. BABUŠKA \& J. E. OSBORN, "Estimates for the errors in eigenvalue and eigenvector approximation by Galerkin methods, with particular attention to the case of multiple eigenvalues," SIAM J. Numer. Anal., v. 24, 1987, pp. 1249-1276.

4. I. BABUŠKA \& J. OSBORN, "Eigenvalue problems," in Handbook of Numerical Analysis (P. G. Ciarlet and J. L. Lions, eds.), North-Holland, Amsterdam. (To appear.)

5. JU. M. BEREZANSKIĬ, Expansions in Eigenfunctions of Selfadjoint Operators, Naukova Dumka, Kiev, 1965; English transl., Transl. Math. Monos., vol. 17, Amer. Math. Soc., Providence, R.I., 1968.

6. J. H. BRAMBLE \& J. E. OSBORN, "Rate of convergence estimates for nonselfadjoint eigenvalue problems," Math. Comp., v. 27, 1973, pp. 525-549.

7. F. Chatelin, "La méthode de Galerkin. Ordre de convergence des éléments propres," C. R. Acad. Sci. Paris Sér. A, v. 278, 1974, pp. 1213-1215.

8. F. Chatelin, Spectral Approximations of Linear Operators, Academic Press, New York, 1983.

9. N. Dunford \& J. T. SChWARTZ, Linear Operators, Part II: Spectral Theory, Self Adjoint Operators in Hilbert Space, Wiley-Interscience, New York, 1963.

10. J. Nitsche, "Ein Kriterium für die Quasi-Optimalität des Ritzschen Verfahrens," Numer. Math., v. 11, 1968, pp. 346-348.

11. L. A. OGANESJAN \& L. A. Rukhovets, "Investigation of the convergence rate of variational-difference schemes for elliptic second order equations in a two dimensional domain with a smooth boundary," (Russian), Ž. Vyčisl. Mat. i Mat. Fiz., v. 9, 1969, pp. 1102-1120; English transl., U.S.S.R. Comput. Math. and Math. Phys., v. 9, no. 5, 1969, pp. 158-183.

12. J. G. PIERCE \& R. S. VARGA, "Higher order convergence results for the Rayleigh-Ritz method applied to eigenvalue problems: II. Improved error bounds for eigenfunctions," Numer. Math., v. 19, 1972, pp. $155-169$.

13. H. F. WEINBERGER, "Error bounds in the Raleigh-Ritz approximation of eigenvectors," $J$. Res. Nat. Bur. Standards, v. 64B, 1960, pp. 217-225. 